\title{
Measurement of the inclusive production cross sections for forward jets and for dijet events with one forward and one central jet in pp collisions at $\sqrt{s}=7 \mathrm{TeV}$
}

\section{The CMS collaboration}

E-mail: cms-publication-committee-chair@cern.ch

ABSTRACT: The inclusive production cross sections for forward jets, as well for jets in dijet events with at least one jet emitted at central and the other at forward pseudorapidities, are measured in the range of transverse momenta $p_{\mathrm{T}}=35-150 \mathrm{GeV} / c$ in proton-proton collisions at $\sqrt{s}=7 \mathrm{TeV}$ by the CMS experiment at the LHC. Forward jets are measured within pseudorapidities $3.2<|\eta|<4.7$, and central jets within the $|\eta|<2.8$ range. The differential cross sections $\mathrm{d}^{2} \sigma / \mathrm{d} p_{\mathrm{T}} \mathrm{d} \eta$ are compared to predictions from three approaches in perturbative quantum chromodynamics: (i) next-to-leading-order calculations obtained with and without matching to parton-shower Monte Carlo simulations, (ii) PYTHIA and HERWIG parton-shower event generators with different tunes of parameters, and (iii) CASCADE and HEJ models, including different non-collinear corrections to standard single-parton radiation. The single-jet inclusive forward jet spectrum is well described by all models, but not all predictions are consistent with the spectra observed for the forward-central dijet events.

KEYwORDS: Hadron-Hadron Scattering

ARXIV EPRINT: 1202.0704 


\section{Contents}

1 Introduction 1

2 Experimental setup $\quad 3$

3 Data selection 3

4 Jet reconstruction 4

$5 \quad$ Jet energy corrections 4

6 Determination of jet cross sections and systematic uncertainties 6

7 Results and comparison to theory $\quad 8$

7.1 Theoretical predictions 8

$\begin{array}{ll}7.2 & \text { Inclusive forward spectrum } \\ \end{array}$

$\begin{array}{lll}7.3 & \text { Forward-central dijet spectra } & 12\end{array}$

$\begin{array}{lll}8 & \text { Summary } & 15\end{array}$

$\begin{array}{ll}\text { The CMS collaboration } & 21\end{array}$

\section{Introduction}

Jet production in hadron-hadron collisions is sensitive to the nature of the underlying parton-parton scattering processes, to the details of parton radiation, as well as to the parton distribution functions (PDF) of the colliding hadrons [1]. The jet cross sections at large transverse momenta $\left(p_{\mathrm{T}}\right)$ measured at the Large Hadron Collider (LHC) $[2,3]$ as well as at previous colliders [4, 5], are well described over several orders of magnitude by perturbative quantum chromodynamics (pQCD). However, the measurements are often limited to central pseudorapidities $(|\eta| \lesssim 3)$, with $\eta=-\log [\tan (\theta / 2)]$ where $\theta$ is the polar angle of the jet with respect to the beam axis. In this region of phase space, the momentum fractions $x_{1}$ and $x_{2}$ of the incoming partons are of the same magnitude. Jets emitted at small polar angles $(|\eta| \gtrsim 3)$ usually arise from collisions between partons of significantly different momentum fractions $x_{2} \ll x_{1}$, and thereby probe regions of PDF with contributions from small as well as large $x$ values, which, especially for gluons, are less well constrained by deep-inelastic scattering data [6]. In the phase space considered in this paper, gluons participate in about $80 \%$ of the partonic interactions that lead to forward jet production, with paired parton momentum fractions of the order of $x_{2} \approx 10^{-4}$ and $x_{1} \approx 0.2$ [7]. 
The type of dijet final states studied in this analysis also provides information on multi-parton production processes with large separations in pseudorapidity whose theoretical description involves multiple scales and possibly large logarithmic contributions. Such event topologies may show deviations from the parton radiation patterns expected from the standard Dokshitzer-Gribov-Lipatov-Altarelli-Parisi (DGLAP) evolution equations [811], as modelled in the approaches of e.g. Balitski-Fadin-Kuraev-Lipatov (BFKL) [12-14], Ciafaloni-Catani-Fiorani-Marchesini (CCFM) [15-18], or gluon saturation [19]. Understanding the dynamics of forward jet production, either with or without accompanying central jets, is also essential for modelling multijet backgrounds at the LHC, e.g. in Higgs boson searches in channels involving vector-boson fusion [20] or requiring a central-jet veto [21], as well as in extracting vector-boson scattering cross sections [22].

The Compact Muon Solenoid (CMS) detector provides a calorimetric coverage to study jet production over a range of jet pseudorapidities as large as $\Delta \eta \approx 10$ which has not been reached heretofore. The study presented here considers the measurement of central and forward jets with maximum rapidity separations of $\Delta \eta \approx 6$ similar to a recent ATLAS study [23]. Here, the inclusive production of forward jets, as well as that of forward jets produced in conjunction with central jets, is studied in data collected with the CMS detector at the LHC during 2010 in proton-proton (pp) collisions at a centre-of-mass energy of $7 \mathrm{TeV}$. The distributions of interest include the single-jet inclusive differential cross section $\mathrm{d}^{2} \sigma / \mathrm{d} p_{\mathrm{T}} \mathrm{d} \eta$ for forward jets, as well as the differential cross sections $\mathrm{d}^{2} \sigma / \mathrm{d} p_{\mathrm{T}}^{f} \mathrm{~d} \eta^{f}$ and $\mathrm{d}^{2} \sigma / \mathrm{d} p_{\mathrm{T}}^{c} \mathrm{~d} \eta^{c}$ for the simultaneous production of at least one forward jet $(f)$ in conjunction with at least one central jet $(c)$. The axis of the forward jet is required to be in the fiducial acceptance of the hadron forward calorimeters $(3.2<|\eta|<4.7)$, and that of the central jet within $|\eta|<2.8$. The concurrent measurement of at least one jet in both $\eta$ regions is referred to as "dijet" in the following.

The final jet spectra are fully corrected to the level of stable particles (lifetime $\tau$ with $c \tau>10 \mathrm{~mm}$ ) coming out from the proton-proton interaction (which we will refer to as "particle-level" hereafter), and compared with predictions from several Monte Carlo (MC) event generators, such as PYTHIA 6 (version 6.422) [24], PYTHIA 8 (version 8.135) [25], HERWIG 6 (version 6.510.3) [26] + JimmY [27], and HERWIG++ (version 2.3) [28]. The data are also compared to next-to-leading-order (NLO) pQCD predictions obtained either with NLOJET $++[29,30]$ corrected for non-perturbative effects, or with the POWHEG package [31] which implements a matching to PYTHIA or HERWIG parton showers. In addition, the measured distributions are compared to results from the CASCADE (version 2.2.04) [32, 33] and HEJ $[34,35]$ programs. CASCADE includes parton radiation from QCD evolution in $1 / x$ and HEJ includes extra contributions from wide-angle gluon radiation, that are not provided in the other models.

This paper is organised as follows. Sections 2 and 3 describe the experimental apparatus and the data sample used in the analysis. Jet reconstruction and energy corrections are presented in sections 4 and 5, respectively. The results and their associated uncertainties, discussed in section 6 , are compared to theoretical expectations in section 7 , and the conclusions are summarised in section 8 . 


\section{Experimental setup}

The CMS detector is described in ref. [36]. Only the detector systems used in this analysis are discussed hereafter. The central feature of the CMS detector is a superconducting solenoid that provides an axial magnetic field of $3.8 \mathrm{~T}$ parallel to the beam axis. Charged particle trajectories are measured using silicon pixels and strip trackers that cover the pseudorapidity region $|\eta|<2.5$. An electromagnetic crystal calorimeter (ECAL) and a brass/scintillator hadron calorimeter (HCAL) surround the tracking volume and cover $|\eta|<3.0$. A forward quartz-fibre Cherenkov hadron calorimeter (HF) extends the coverage to $|\eta|=5.2$.

The relevant detector components for the reconstruction of jets in this work are the ECAL and HCAL central calorimeters [37, 38], as well as the HF forward calorimeters [39]. The ECAL cells are grouped in quasi-projective towers of granularity in pseudorapidity and azimuthal angle of $\Delta \eta \times \Delta \phi=0.0174 \times 0.0174$ in the barrel $(|\eta|<1.5)$, and of $0.05 \times 0.05$ in the endcap $(1.5<|\eta|<3.0)$. The HCAL has a tower granularity as small as $\Delta \eta \times$ $\Delta \phi=0.087 \times 0.087$. The HCAL, when combined with the ECAL, measures jets with a resolution $\Delta E / E \approx 100 \% / \sqrt{E(\mathrm{GeV})} \oplus 5 \%$. The HF calorimeters consist of steel absorbers containing embedded radiation-hard quartz fibres, located at $\pm 11.2 \mathrm{~m}$ from the centre of the CMS detector, and cover the region $2.9<|\eta|<5.2$. Half of the fibres run over the full longitudinal depth of the absorber, while the other half start at a depth of $22 \mathrm{~cm}$ from the front face of each detector. The segmentation of the HF calorimeters is $0.175 \times 0.175$, except for $|\eta|>4.7$, where it is $0.175 \times 0.35$. The HF energy resolution is $\sim 200 \% / \sqrt{E(\mathrm{GeV})}$.

\section{Data selection}

For online selection, CMS uses a two-level trigger system consisting of a Level-1 and a High Level Trigger (HLT). The HLT searches for jets using an iterative cone algorithm [40, 41] of radius $R=\sqrt{(\Delta \eta)^{2}+(\Delta \phi)^{2}}=0.5$. Events for the inclusive forward-jet analysis were selected with a trigger requiring a minimum jet transverse energy of $15 \mathrm{GeV}$ within $|\eta|<5.2$, while the events used in the dijet measurement were taken with a dijet trigger requiring two jets with summed calorimeter transverse energy above $30 \mathrm{GeV}$ also within $|\eta|<5.2$. The total data sample collected at luminosities of about $10^{30} \mathrm{~cm}^{-2} \mathrm{~s}^{-1}$ corresponds to an integrated luminosity of $(3.14 \pm 0.14) \mathrm{pb}^{-1}$. Trigger efficiencies are determined from the ratio of the yield of events containing either forward or forward-central jets that pass the HLT requirements over the yield of events that pass the minimum-bias and low-threshold $(6 \mathrm{GeV})$ jet-monitor triggers. In all cases, the HLT is fully efficient for single jets with calibrated $p_{\mathrm{T}}>35 \mathrm{GeV} / c$.

All events are required to have a primary vertex reconstructed from at least 5 tracks, consistent with the known transverse position of the beams and within $\pm 24 \mathrm{~cm}$ of the centre of the detector along the longitudinal direction. Events are further filtered out in the pixel detector, by requiring more than $25 \%$ well-reconstructed tracks in events with 10 or more tracks [42]. Events with anomalous noise in HF calorimeters, e.g. due to energetic charged particles that interact in the window of the HF photo-multipliers, are flagged with 
different algorithms and rejected. These selection criteria reject non-collision and beamrelated backgrounds and are highly efficient $(\sim 100 \%)$ for the final states in this analysis.

\section{Jet reconstruction}

The anti- $k_{T}$ jet clustering algorithm $[43,44]$ is used to reconstruct forward and central jets with a distance parameter $R=0.5$. The inputs to the clustering correspond to depositions of energies in calorimeter cells and their angles relative to the beam axis. A four-momentum is associated to each jet by summing the energy of the cells above a given threshold, assuming zero mass for each cell deposit, with momentum components specified by the angles of each cell relative to the point of interaction given by the main event vertex [45]. In the central region, jets are obtained from signals in calorimeter towers with energies in at least one HCAL cell, and from their geometrically corresponding ECAL crystals. In the forward region, jets are reconstructed using Cherenkov-light signals collected in both the HF short and long quartz readout fibres.

The central and forward jet regions are defined, respectively, as $|\eta|<2.8$ and $3.2<$ $|\eta|<4.7$, where $\eta$ corresponds to the reconstructed jet axis vector applied on the interaction point. Both $|\eta|$ ranges guarantee full jet reconstruction within the maximum calorimeters limits taking into account the jet size parameter of $R=0.5$. All jets are required to have a transverse momentum above $p_{\mathrm{T}}=35 \mathrm{GeV} / c$. If more than one jet is present in either the central or forward region, the one with highest $p_{\mathrm{T}}$ is considered in this analysis. Central jets are required to satisfy the calorimeter quality criteria of ref. [45]. In the HF calorimeter, the applied jet quality selections remove unphysical energy depositions. These criteria require each jet to have a minimum ( $p_{\mathrm{T}}$-dependent) number of $\mathrm{HF}$ cells clustered into a jet, and the fraction of the electromagnetic to total jet energies to be above a parameterised threshold.

\section{Jet energy corrections}

The jet $p_{\mathrm{T}}$ spectra reconstructed from the calorimeter energies are corrected to account for the following systematic effects: (i) $p_{\mathrm{T}^{-}}$and $\eta$-dependent response of the calorimeters, and possible overlap with other proton-proton interactions (pileup), and (ii) an "unfolding" of the impact of the jet energy resolution on the migration of events across $p_{\mathrm{T}}$ bins, and thereby correct the measured spectrum to the particle-level through comparison with MC events, as discussed below.

The reconstructed jet energy scale (JES) is first calibrated using data based on balancing the $p_{\mathrm{T}}$ values in dijet and in photon-jet events, as well as from MC simulations [45]. The ensuing JES corrections adjust the energies according to the relative $\eta$ and $p_{\mathrm{T}}$ dependencies of the response of the ECAL, HCAL and HF calorimeters. These corrections, with values from 1.0 to 1.2 for HF, set the absolute energies to their calibrated JES values and also account for the extra pileup energy. The latter effect is very small in this analysis which is mostly based on data collected with un-prescaled low- $p_{\mathrm{T}}$ jet triggers during the early running of the LHC with an average of $\sim 2.2$ collisions per colliding pair of proton bunches. 


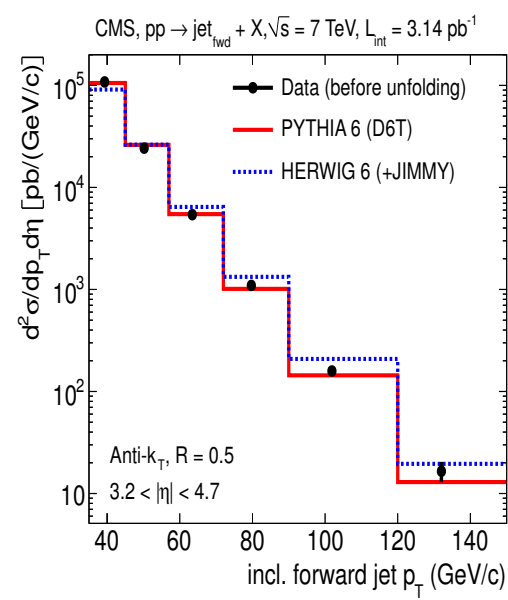

(a)

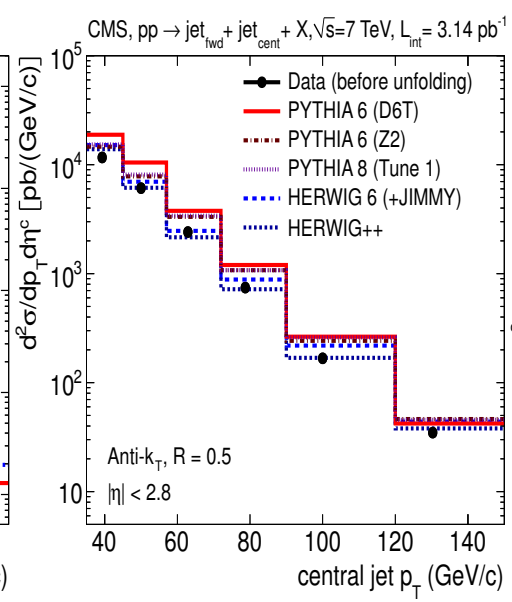

(b)

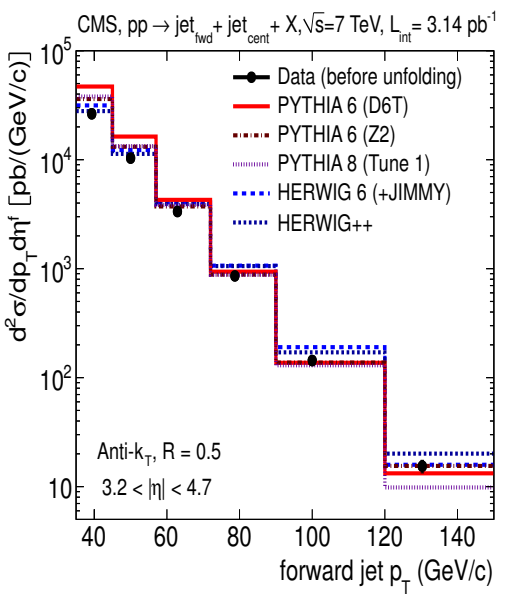

(c)

Figure 1. Measured differential cross sections for jets as a function of $p_{\mathrm{T}}$, before unfolding the energy resolution (black dots), compared to detector-level MC simulations generated with different versions of PYTHIA and HERWIG (histograms) for (a) inclusive forward jets, and for (b) central and (c) forward jets in dijet events.

Figure 1 shows the reconstructed $p_{\mathrm{T}}$ spectrum for: (a) inclusive forward jets, and (b) central and (c) forward jets in dijet events. These are compared to MC events passed through full detector simulation based on GEANT [46], and analysed in the same way as the data. The data shown are calibrated through the JES normalisation, but not unfolded. The cross sections in each interval of $\eta$ and $p_{\mathrm{T}}$ are divided by their bin-widths. With the simulated events normalised to the integrated luminosity used in this analysis, all the models considered provide inclusive forward jet spectra consistent with the data, but tend to overestimate the absolute cross sections measured in forward-central dijet events as discussed later.

The second correction (unfolding) of the measured jet spectrum is applied to account for the finite energy resolution of the calorimeters. Although the bin size of the presented $p_{\mathrm{T}}$ distributions equals or exceeds the experimental resolution, the combination of a steeply falling $p_{\mathrm{T}}$-spectrum and calorimeter resolutions leads to migration of events across bins that can distort the distribution in $p_{\mathrm{T}}$. At central rapidities, the relative resolution in jet $p_{\mathrm{T}}$, obtained from studies of $p_{\mathrm{T}}$ imbalances in dijet events in data and in MC simulations, changes from 15 to $8 \%$ in the $p_{\mathrm{T}}$ range of interest. For forward jets, the relative resolution in $p_{\mathrm{T}}$, derived from full-simulation studies and confirmed by the momentum imbalance in dijet data, is below $12 \%$ for $p_{\mathrm{T}}>35 \mathrm{GeV} / c$. In the $p_{\mathrm{T}} \approx 35-80 \mathrm{GeV} / c$ range, the transverse momentum resolution for HF jets is better than for central jets because of the $\cosh (\eta)$ boost factor for the total energy deposited in the calorimeter at forward rapidities [7]. Two methods are used to account for the bin-migration effect:

(i) Exploiting the fact that MC simulations (figure 1) reproduce the $p_{\mathrm{T}}$-dependence of the inclusive forward-jet spectrum, and that the simulated spectra for dijet events can be 
re-weighted to match the shape of the measured distributions, the MC samples are used to study the bin-to-bin migrations. The correction factors have also been crosschecked by inverting the response matrix obtained from the MC information, albeit with limited statistics, through the application of different unfolding algorithms [47].

(ii) The measured jet $p_{\mathrm{T}}$ spectrum is fitted to some parameterised function $f\left(p_{\mathrm{T}}\right)[48,49]$, the result of which can be smeared using the known (Gaussian) jet resolutions $[2,45]$. The parameters of the model are then defined by fitting the smeared transverse $p_{\mathrm{T}}$ spectrum $F\left(p_{\mathrm{T}}\right)$ to the measured $f\left(p_{\mathrm{T}}\right)$, and using the ratio of both distributions for the final correction [2].

The difference between the results of the two methods is below $10 \%$ for all $p_{\mathrm{T}}$ bins. The factors obtained with the MC method are used to correct the mean values of $p_{\mathrm{T}}$, while the results from the fits are used to assess the associated systematic uncertainties. The MC-based method also takes into account various final-state effects, such as hadronisation and particle decays, which affect the final energy clustered into jets. The corresponding bin-by-bin factors thus fully correct the jet spectrum from the detector to the particle levels via the factor

$$
C_{\text {had }}\left(p_{\mathrm{T}}, \eta\right)=\frac{N_{\text {had }}^{\mathrm{MC}}\left(p_{\mathrm{T}}, \eta\right)}{N_{\mathrm{det}}^{\mathrm{MC}}\left(p_{\mathrm{T}}, \eta\right)},
$$

where $N_{\mathrm{had}}^{\mathrm{MC}}\left(p_{\mathrm{T}}, \eta\right)$ and $N_{\mathrm{det}}^{\mathrm{MC}}\left(p_{\mathrm{T}}, \eta\right)$ are the jet event yields determined after hadronisation and after full simulation, respectively. The factor $N_{\text {had }}^{\mathrm{MC}}\left(p_{\mathrm{T}}, \eta\right)$ is obtained by averaging the predictions from PYTHIA 6 with HERWIG $6+$ JIMMY, which provide different modelling of parton-to-hadron processes, one based on string and the other on cluster fragmentation, respectively. The unfolding correction factors obtained for the two event generators differ by less than 5\%, as shown in the left panel of figure 2. (The average of the two MC predictions is shown in the two right panels.) The hatched band in all panels indicates the uncertainty obtained by changing the jet $p_{\mathrm{T}}$ resolution by $\pm 10 \%$, and covers the range of differences found for the two methods of unfolding the data.

\section{Determination of jet cross sections and systematic uncertainties}

The final data sample contains events with at least one forward jet or at least a forward and a central jet satisfying the selections described in section 3 . The corresponding numbers of events, $N_{\text {evts }}$, are binned into a differential inclusive jet cross section as a function of $\eta$ and $p_{\mathrm{T}}$ :

$$
\frac{\mathrm{d}^{2} \sigma}{\mathrm{d} p_{\mathrm{T}} \mathrm{d} \eta}=\frac{C_{\text {had }}}{\mathcal{L} \cdot \varepsilon_{t}} \cdot \frac{N_{\text {evts }}}{\Delta p_{\mathrm{T}} \cdot \Delta \eta} .
$$

The factor $C_{\text {had }}$ accounts for bin-to-bin migrations due to $p_{\mathrm{T}}$ resolution and detector to particle corrections, eq. (5.1), $\mathcal{L}$ is the total integrated luminosity, $\varepsilon_{t}$ is the efficiency of the jet trigger, and $\Delta p_{\mathrm{T}}$ and $\Delta \eta$ are the sizes of the bins in $p_{\mathrm{T}}$ and $\eta$, respectively. The jet triggers have a $\varepsilon_{t}=100 \%$ efficiency for all $p_{\mathrm{T}}$ and $\eta$ values considered in this paper with a negligible contribution to the total systematic uncertainty. 


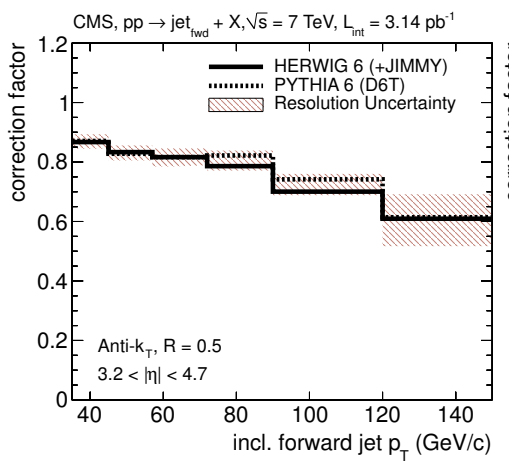

(a)

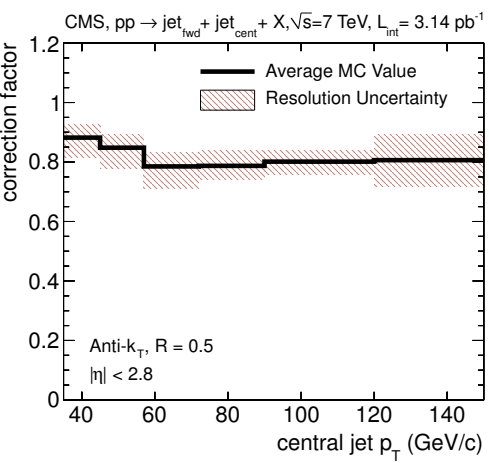

(b)

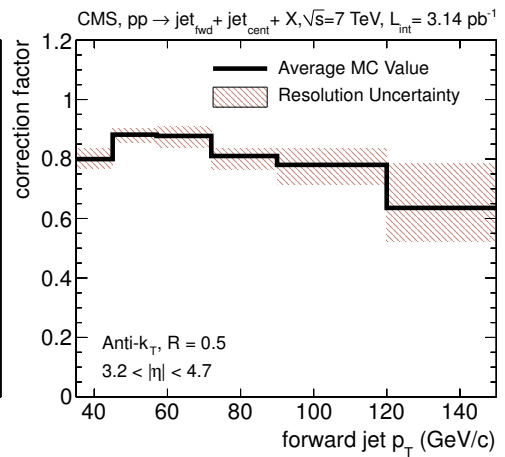

(c)

Figure 2. The bin-by-bin unfolding correction factors as a function of $p_{\mathrm{T}}$ for (a) inclusive forward jets, and for (b) central and (c) forward jets in dijet events. Panel (a) shows the individual correction factors obtained with PYTHIA 6 and HERWIG 6, while (b) and (c) show the average values obtained for the two MC generators (black histograms). The hatched band represents the uncertainties assigned to the correction factors as described in the text.

There are three primary sources of systematic uncertainty in the jet cross sections measurements:

(i) Jet energy scale in the calorimeters. At forward rapidities, the HF calorimeter has a JES calibration uncertainty that varies between 3 and $6 \%$, depending on the $p_{\mathrm{T}}$ and $\eta$ of the reconstructed jet [45]. This uncertainty must be convoluted with that associated with a $\sim 0.8 \mathrm{GeV}$ energy shift per pileup-event due to the presence of other hadrons around the forward jet axis. The JES uncertainties of the central calorimeters have typical values between 2.5 and $3.5 \%$ [45]. The uncertainty from pileup energy has been studied by comparing central jet $p_{\mathrm{T}}$ distributions with and without the requirement to have only one primary vertex in the event. The central jet $p_{\mathrm{T}}$ spectra under these two conditions are found to differ by less than $5 \%$. The JES uncertainties, propagated to the steeply falling jet spectra (inverse power-law $p_{\mathrm{T}}$ distributions with exponent of $n \approx 5$ ), translate into uncertainties of the order of $\pm(20-30) \%$ in the final forward and central jet cross sections.

(ii) Unfolding procedure and $p_{\mathrm{T}}$ resolution $\left(C_{\text {had }}\right.$ factor). The $\pm 10 \%$ uncertainty on the jet $p_{\mathrm{T}}$ resolution (figure 2) translates into an uncertainty of 3 to $6 \%$ (increasing with $\left.p_{\mathrm{T}}\right)$ on the final cross sections. An additional uncertainty of $3 \%$, from the model dependence of the correction factors defined by the difference between the PYTHIA 6 and HERWIG 6 generators used to unfold the cross sections, is added in quadrature.

(iii) Luminosity. The uncertainty of the integrated pp luminosity results in a $4 \%$ uncertainty on the overall normalisation of the spectra [50].

In all $p_{\mathrm{T}}$ bins of the measured cross sections, the statistical uncertainty (of the order of $1-2 \%$ in the low $p_{\mathrm{T}}$ bin and $5-10 \%$ in the highest) is small relative to the systematic uncertainty obtained by adding all contributions in quadrature. The latter amounts to 


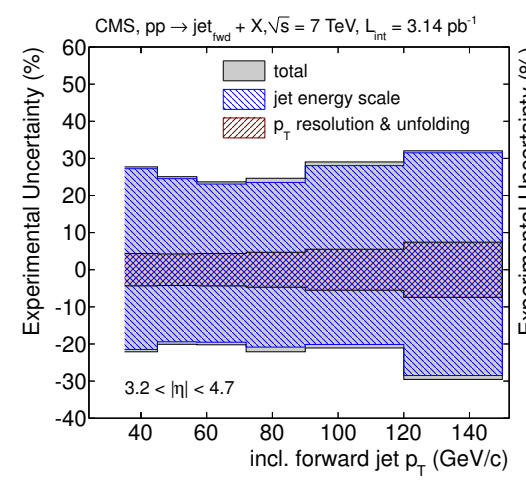

(a)

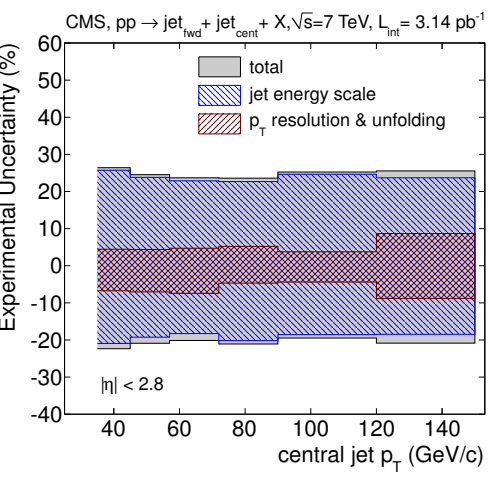

(b)

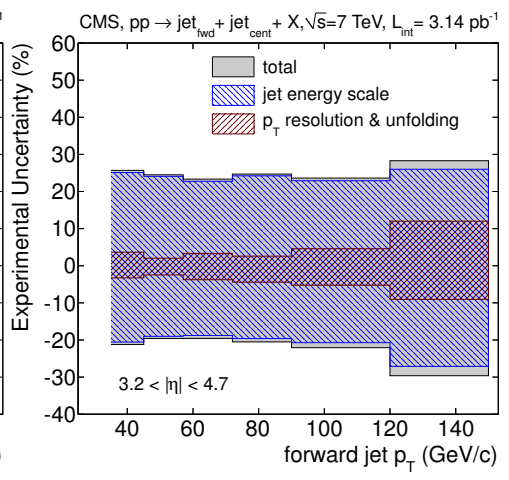

(c)

Figure 3. Systematic uncertainties as a function of jet $p_{\mathrm{T}}$ for (a) inclusive forward production, and for (b) central and (c) forward jet spectra in dijet events. The outer limits of the grey areas show the overall uncertainties, from adding in quadrature uncertainties from the JES, the unfolding and the luminosity.

\begin{tabular}{|cccc|}
\hline $\begin{array}{c}p_{\mathrm{T}} \text { bin (centre) } \\
\mathrm{GeV} / c\end{array}$ & $\begin{array}{c}\frac{\mathrm{d}^{2} \sigma}{\mathrm{d} p_{\mathrm{T}} \mathrm{d} \eta} \\
\mathrm{pb} /(\mathrm{GeV} / c)\end{array}$ & $\begin{array}{c}\frac{\mathrm{d}^{2} \sigma}{\mathrm{d} p_{\mathrm{T}}^{c} \mathrm{~d} \eta^{c}} \\
\mathrm{pb} /(\mathrm{GeV} / c)\end{array}$ & $\begin{array}{c}\frac{\mathrm{d}^{2} \sigma}{\mathrm{d} p_{\mathrm{T}}^{f} \mathrm{~d} \eta^{f}} \\
\mathrm{pb} /(\mathrm{GeV} / c)\end{array}$ \\
\hline $35-45(39.3)$ & $\left(89 \pm 0.2_{-19}^{+24}\right) \times 10^{3}$ & $\left(10 \pm 0.1_{-2.2}^{+2.6}\right) \times 10^{3}$ & $\left(21 \pm 0.2_{-0.5}^{+5.4}\right) \times 10^{3}$ \\
$45-57(50.2)$ & $\left(20 \pm 0.1_{-3.9}^{+4.9}\right) \times 10^{3}$ & $\left(5.2 \pm 0.07_{-1.0}^{+1.2}\right) \times 10^{3}$ & $\left(9.2 \pm 0.1_{-1.8}^{+2.2}\right) \times 10^{3}$ \\
$57-72(63.2)$ & $\left(4.4 \pm 0.04_{-0.9}^{+1.0}\right) \times 10^{3}$ & $\left(1.9 \pm 0.03_{-0.4}^{+0.4}\right) \times 10^{3}$ & $\left(2.9 \pm 0.06_{-0.6}^{+0.7}\right) \times 10^{3}$ \\
$72-90(79.2)$ & $880 \pm 10_{-180}^{+200}$ & $590 \pm 20_{-120}^{+130}$ & $690 \pm 30_{-140}^{+170}$ \\
$90-120(101.0)$ & $115 \pm 4_{-25}^{+40}$ & $135 \pm 6_{-25}^{+33}$ & $110 \pm 8_{-25}^{+25}$ \\
$120-150(132.0)$ & $10 \pm 1.2_{-3}^{+3}$ & $28 \pm 3_{-5}^{+7}$ & $10 \pm 2.3_{-3}^{+3}$ \\
\hline
\end{tabular}

Table 1. Measured $p_{\mathrm{T}}$-dependent differential cross sections for inclusive forward jets (second column), and for central (third column) and forward (last column) jets in dijet events. The first (second) uncertainty reflects the statistical (systematic) contribution.

$\sim 30 \%$ and is dominated by the uncertainty on the calibration of the jet energy scale. The different contributions to the systematic uncertainty are shown as a function of jet $p_{\mathrm{T}}$ in figure 3 for the three $p_{\mathrm{T}}$ distributions of interest. The grey areas show the total uncertainty, while the two hatched areas indicate the uncertainties on the JES and the unfolding procedure. Table 1 tabulates the measured, fully-corrected $p_{\mathrm{T}}$-differential jet cross sections and their associated uncertainties.

\section{Results and comparison to theory}

\subsection{Theoretical predictions}

The measured differential jet cross sections are compared to predictions from different pQCD approaches: (i) general-purpose event generators PYTHIA 6 (version 6.422) with 
D6T and Z2 tunes [24, 51], PYthia 8 (version 8.135) with Tune 1 [25], HeRWig 6 (version 6.510.3) [26] with underlying-event modelled with JIMMY [27], and HERWIG++ (version 2.3) [28], (ii) NLO calculations obtained with the POWHEG package [52] (matched with PYTHIA and HERWIG parton showers) as well as with NLOJET ++ [29] within the FASTNLO [53] package, for different sets of parton densities, and (iii) the CASCADE (version 2.2.04) $[54,55]$ and HEJ $[34,35]$ codes.

The PYTHIA and HERWIG Monte Carlo event generators are based on standard collinear (DGLAP) evolution, where the parton shower can be developed by ordering the parton splittings in $p_{\mathrm{T}}$ (in the Z2 tune, in Tune 1, or in combination with the POWHEG NLO generator) or in virtuality $Q^{2}$ (in the D6T tune). HERWIG uses angular ordering for shower evolution. The PYTHIA 6 and HeRWig 6 event generators use the CTEQ6L PDF [56], whereas CTEQ5L [57] has been used for PYTHIA 8, and the MRST2001 PDF [58] for HERWIG ++ . The default NLOJET ++ calculation is run with CT10 [59], and POWHEG is run with the CTEQ6M PDF [56] plus PYThia 6 (Perugia 0 tune [60]) and HeRwig 6 for the parton showering and hadronisation. The default renormalisation and factorisation scales have been set to $\mu_{\mathrm{r}}=\mu_{\mathrm{f}}=p_{\mathrm{T}}$ for both NLO calculations. The CASCADE Monte Carlo program, based on resummation of leading logarithms in virtuality $Q^{2}$ and in parton momentum fraction $x$, as implemented in the CCFM evolution equations, uses the Set-A unintegrated parton distributions [61] and a cut on the $p_{\mathrm{T}}$ of the matrix-element partons of $14 \mathrm{GeV} / c$. The HEJ event generator uses the MSTW2008NLO PDFs [62] and provides, at parton level, an all-order description of the dominant radiative corrections for hard, wide-angle emissions.

Before comparing the data to parton-level predictions such as NLOJET ++ or HEJ, the uncertainties from non-perturbative (NP) effects must be determined. Such effects include energy lost from the jet in the hadronisation process or "splashed-in" from the underlying event (UE) into the jet, and are estimated as in ref. [63], by comparing the PYTHIA 6 and HERWIG $6+$ JIMMY parton-level spectra with the corresponding particle-level predictions after hadronisation and UE activity. Each MC program has a different way of modelling parton hadronisation and multiparton interactions, that results in different UE characteristics. The NP correction factors amount to 1.10 (1.02) at the lowest (highest) $p_{\mathrm{T}}$ bin considered in this study. Half of the difference between these two predictions, displayed as a function of forward jet $p_{\mathrm{T}}$ in figure 4 , is taken as an estimate of the total systematic uncertainty associated with this NP effect.

For NLO predictions (NLOJET ++ and POWHEG), the uncertainties associated with the PDF and the strong coupling $\alpha_{S}$ can be estimated following the PDF4LHC interim recommendation [64]. The uncertainty on the PDF is estimated from the maximum envelope obtained from the $68 \%$ confidence-level eigenvectors (CL68) of the CT10, MSTW2008 and NNPDF2.1 [65] sets. The uncertainty from the value of the strong coupling $\alpha_{S}$ is derived from separate fits using the CT10 PDF, where $\alpha_{S}\left(M_{Z}\right)$ is changed by \pm 0.002 , and is added in quadrature to the uncertainty on the PDF. The uncertainty associated with higher-order corrections neglected in the NLO calculation has been evaluated by changing the renormalisation and factorisation scales by factors proportional to the jet $p_{\mathrm{T}}$ in the following six combinations: $\left(\mu_{\mathrm{r}}, \mu_{\mathrm{f}}\right)=\left(p_{\mathrm{T}} / 2, p_{\mathrm{T}} / 2\right),\left(p_{\mathrm{T}} / 2, p_{\mathrm{T}}\right),\left(p_{\mathrm{T}}, p_{\mathrm{T}} / 2\right),\left(p_{\mathrm{T}}, 2 p_{\mathrm{T}}\right),\left(2 p_{\mathrm{T}}, p_{\mathrm{T}}\right)$ and $\left(2 p_{\mathrm{T}}, 2 p_{\mathrm{T}}\right)[66]$. Figure 4 (a) shows all the sources of theoretical uncertainty. The NP 


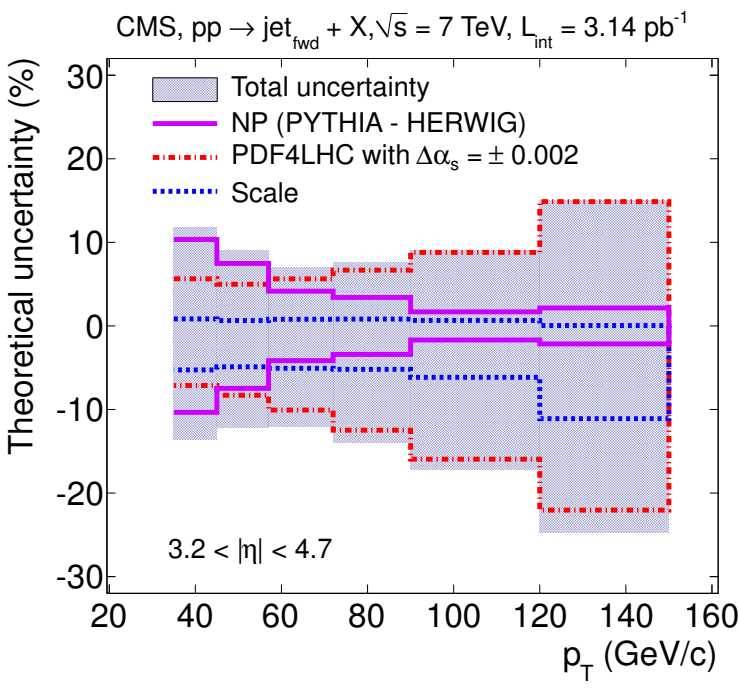

(a)

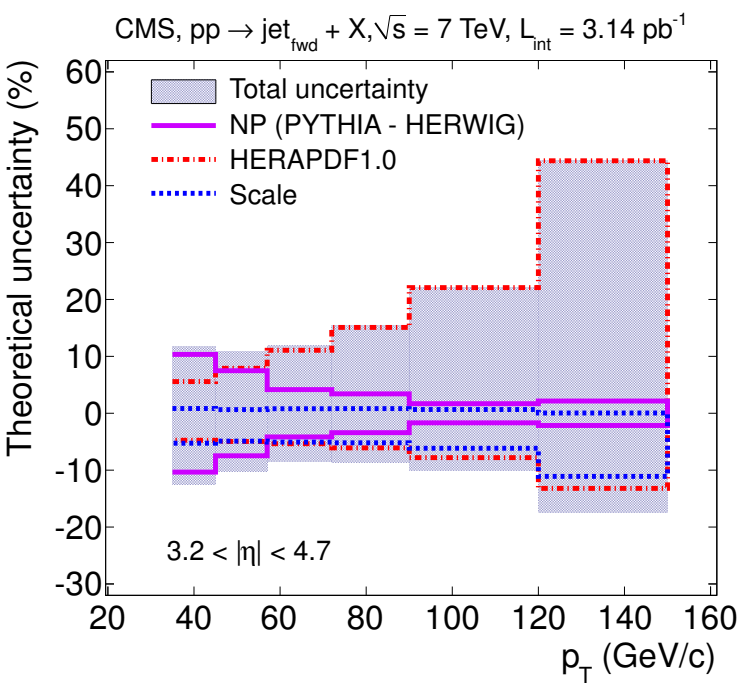

(b)

Figure 4. Uncertainties on the predicted NLO inclusive forward jet spectrum. Plot (a) shows the contributions from non-perturbative effects, choice of PDF and the value of the strong coupling $\alpha_{S}$ (computed with the PDF4LHC prescription), and uncertainties associated with the renormalization and fragmentation scales. Plot (b) shows the uncertainties from NP, PDF and $\alpha_{S}$ (obtained with HERAPDF1.0), and the theoretical scales. Total uncertainties are obtained by adding quadratically the uncertainties on NP, PDF and the scales.

corrections dominate for $p_{\mathrm{T}}<60 \mathrm{GeV} / c$, whereas uncertainties on PDF and $\alpha_{S}$ dominate

above that $p_{\mathrm{T}}$. Scale uncertainties are less important at all transverse momenta. These three sources of uncertainty are added in quadrature into a single band representing the NLO theoretical uncertainty.

An independent cross-check of the uncertainty due to the PDF choice is given in figure 4(b), which shows the same uncertainties for NP and scale, but with the PDF envelope obtained by using the HERAPDF1.0 parton densities [6]. The 33 HERAPDF1.0 PDF eigenvalues correspond to $68 \%$ CL intervals of this PDF that account for experimental, model and parametrisation uncertainties on the fit to HERA data. Two more HERAPDF1.0 fits, with $\alpha_{s}$ changed by \pm 1 standard deviation of the world-average value $(0.1176 \pm 0.002)$ [67], are also checked, and the corresponding effect added in quadrature to the PDF uncertainty. For jets at high $p_{\mathrm{T}}$, this uncertainty is larger than the one obtained with the PDF4LHC prescription, as the HERAPDF1.0 sets have fewer constraints on the gluon density at high$x$ than other globally-fitted PDF, and because HERAPDF also includes extra uncertainties on the initial shape of the parton distributions.

\subsection{Inclusive forward spectrum}

The fully corrected inclusive forward jet cross section as a function of $p_{\mathrm{T}}$ is shown in figure 5(a) compared to the models discussed above. The data points are plotted at the "true" 


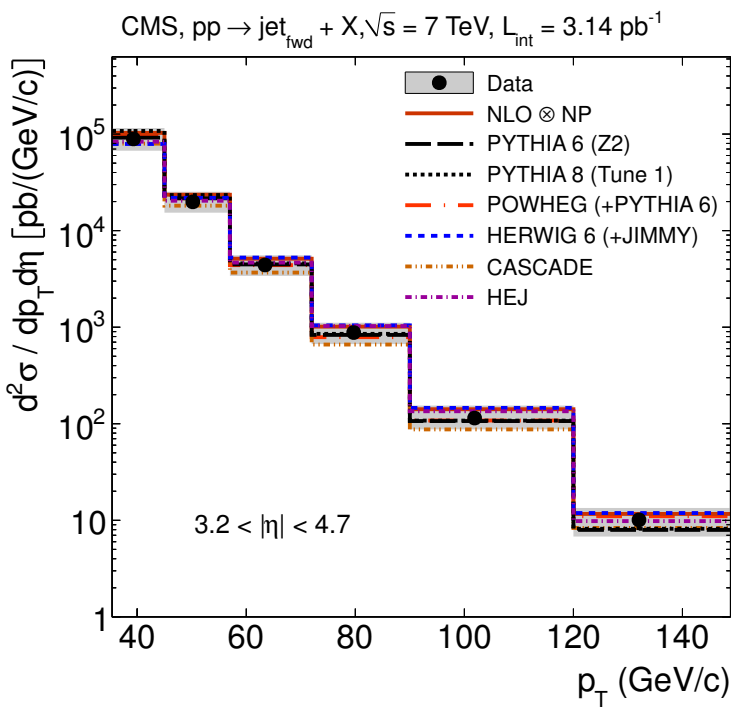

(a)

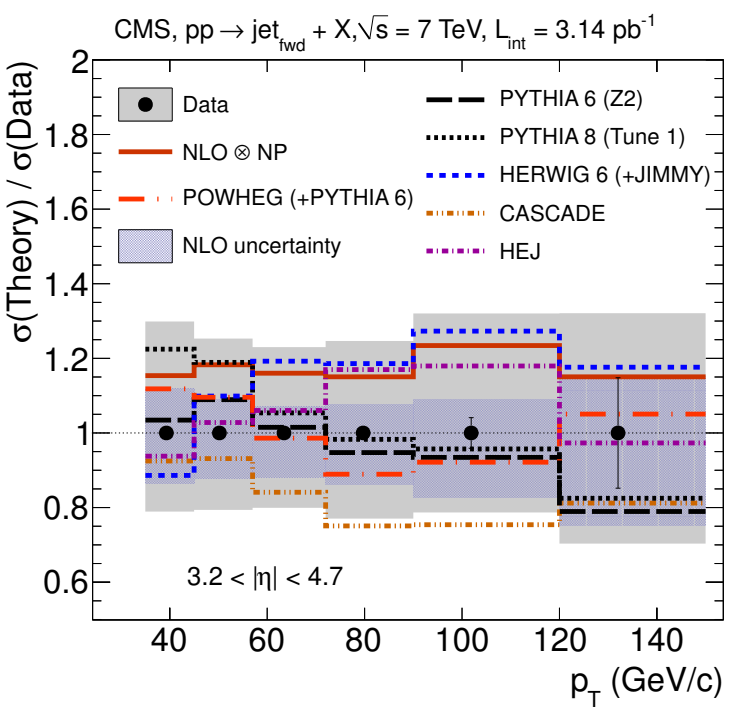

(b)

Figure 5. (a) Inclusive jet cross section at forward pseudorapidities $(3.2<|\eta|<4.7)$, fully corrected and unfolded, compared to particle-level predictions from PYTHIA 6, PYTHIA 8, HERWIG 6 , NLOJET ++ corrected for non-perturbative effects, POWHEG, CASCADE and HEJ. (b) Ratio of theory/data for the forward jet spectrum. The error bars on all data points (which, in (a), are smaller than the size of the markers) reflect just statistical uncertainties, with systematic uncertainties plotted as grey bands. The dark band in (b) shows the theoretical uncertainty on the NLO predictions.

centre of the $p_{\mathrm{T}}$ distribution in that bin [68]. The experimental systematic uncertainty (figure 3 ) is shown as a grey band. Figure 5(b) shows the ratio of theoretical to experimental jet cross sections, including the NLO band of uncertainty (figure 4). Within the theoretical and experimental uncertainties, the predictions are in good agreement with the measurements.

To gauge the sensitivity of the forward jet measurement to the underlying parton densities in the proton, figure 6 shows the NLO predictions compared to the data in the form of bin-by-bin ratios of data to theory (which is used instead of theory/data to improve graphical presentation at high $p_{\mathrm{T}}$ where the reference NLO prediction is not statistically limited). A similar study for jets measured by CMS at central rapidities can be found in ref. [69]. Uncertainties from NP corrections and the renormalisation and factorisation scale variations, common to all theoretical predictions, are added in quadrature and represented by the dashed (magenta) lines around the ratio at unity in figures 6(b) and (c). Uncertainties on individual PDF sets are displayed as bands. To improve the readability, the comparisons to data are performed separately in panel (a) using the central values of all investigated PDF sets relative to CT10, in panel (b) for MSTW2008 and NNPDF2.1, and in panel (c) for HERAPDF1.0 and ABKM09.

All NLO predictions for different PDF are similar and consistent with the data, although they tend to systematically overestimate the central values of the measured forward jet cross sections by $\sim 20 \%$ in all $p_{\mathrm{T}}$ bins. A similar overestimate has been observed for jets at more central pseudorapidities $[2,69]$. 


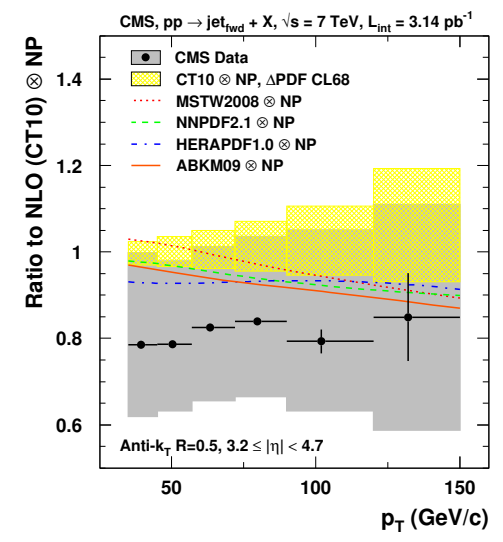

(a)

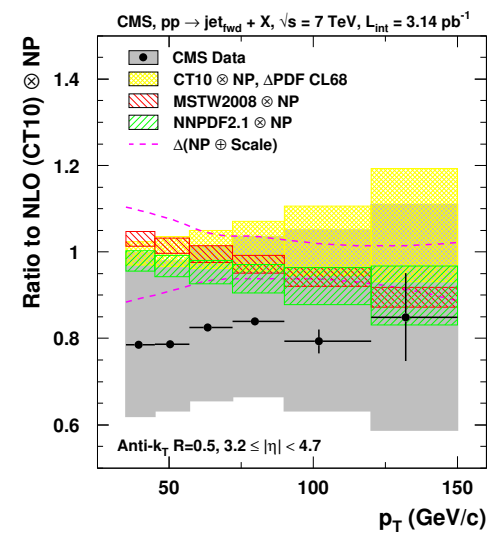

(b)

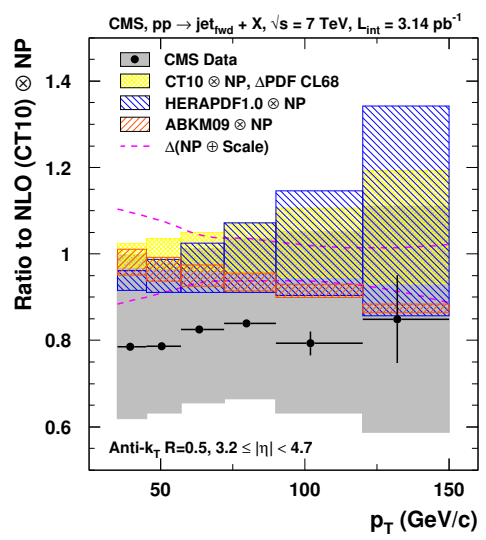

(c)

Figure 6. Ratio of the inclusive forward jet spectrum for data over the NLO predictions using the CT10 PDF, as a function of $p_{\mathrm{T}}$, shown with the statistical (error bars) as well as systematic uncertainties (grey band). Additional predictions are shown in (a) for all the central PDF predictions (curves), (b) for the MSTW2008 and NNPDF2.1 sets, and in (c) for the HERAPDF1.0 and ABKM09 PDF. The corresponding PDF uncertainties are shown as coloured bands around the ratios. Common theoretical uncertainties from choices of scale and non-perturbative corrections are indicated by dashed (magenta) curves in (b) and (c).

\subsection{Forward-central dijet spectra}

The fully corrected $p_{\mathrm{T}}$-dependence of the cross section for the simultaneous production of at least one forward and at least one central jet is presented in figure 7 (a) and (c) for central and in (b) and (d) for forward jets, respectively. The grey bands indicate the systematic uncertainties. The cross sections obtained with PYTHIA 6 (version 6.422) for D6T and Z2 tunes, PYTHIA 8 (version 8.135), POWHEG (using PYTHIA for parton showering and hadronisation), and CASCADE (version 2.2.04) are superimposed on the data in panels (a) and (b), along with those for HERWIG 6 (version 6.510.3), HERWIG++ (version 2.3), POWHEG (using HERWIG for parton showering and hadronisation), and HEJ, shown in panels (c) and (d).

The compatibility of the different models with the measured cross sections is examined through the ratios of predictions to data as a function of jet $p_{\mathrm{T}}$ in figure 8. Most models tend to predict larger values than observed. The HERWIG and HERWIG++ MC event generators that use angular ordering for parton showering appear to be consistent with the data. The other generators, and different tunes, do not describe the data over the full range of $p_{\mathrm{T}}$ values. PYTHiA 8 with Tune 1 and PYTHIA 6 with Tune Z2 ( $p_{\mathrm{T}}$-ordered showering) describe the data better than Tune D6T ( $Q^{2}$-ordered showering). The Z2 parameterisation tuned to the underlying event at the LHC, although reproducing the central jet spectrum more satisfactorily than D6T or PYTHIA 8, still lies well above the data (the same holds true at lower $p_{\mathrm{T}}$ for the forward-jet spectrum). The discrepancy between PYTHIA and data is therefore only partly reduced through changes of the modelling of underlying event and initial- and final-state radiation. 


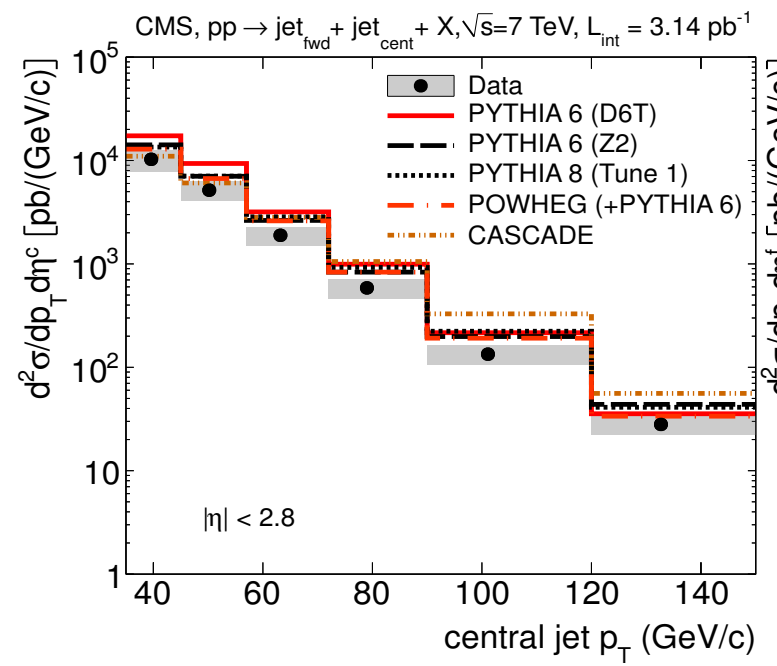

(a)

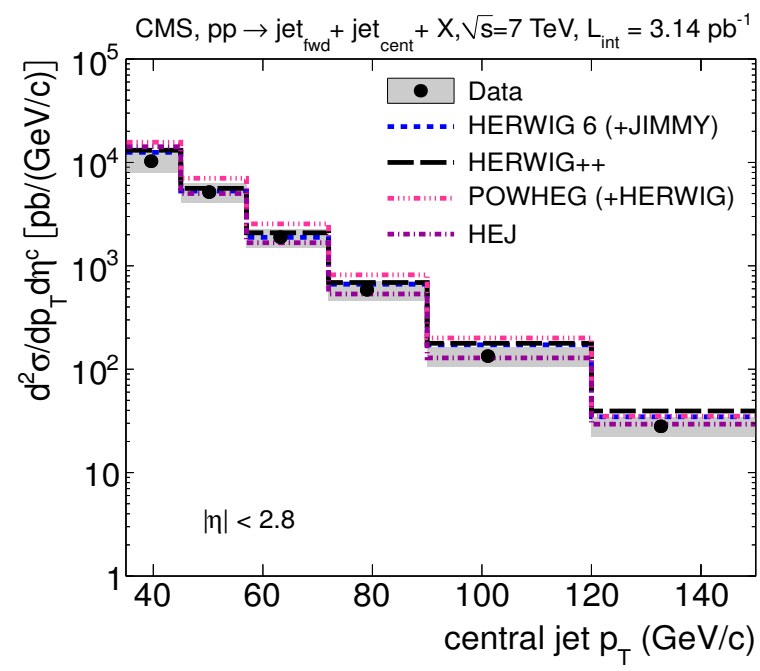

(c)

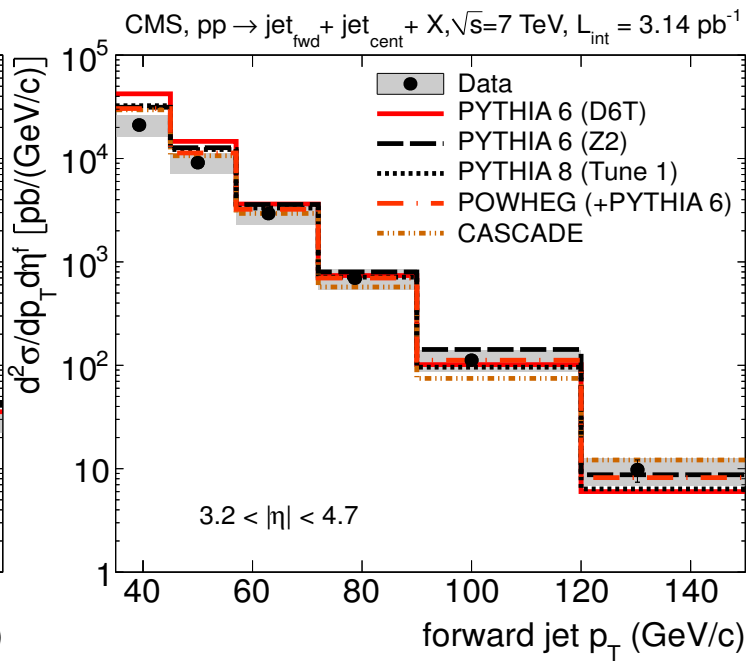

(b)

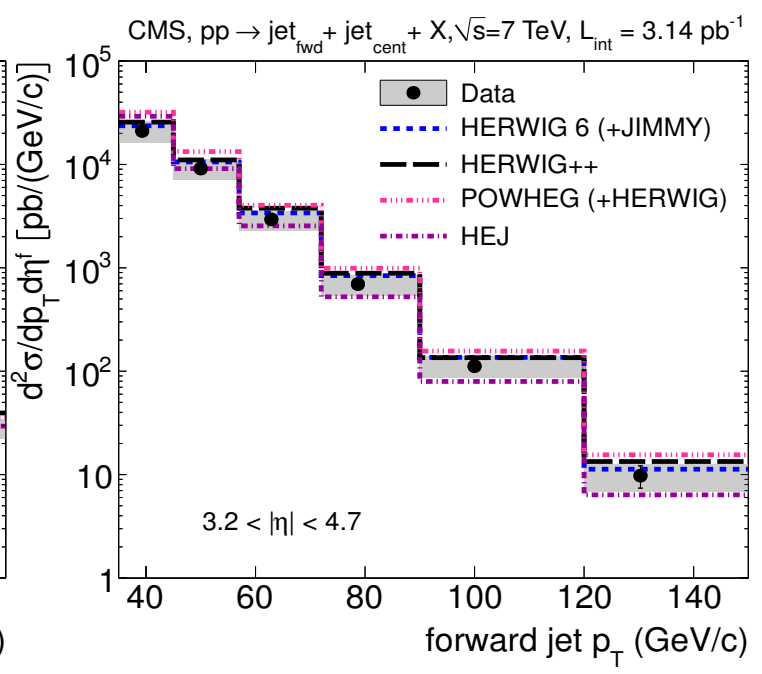

(d)

Figure 7. Differential cross sections as a function of jet $p_{\mathrm{T}}$ for dijet events with at least one central jet ((a) and (c)) and one forward jet ((b) and (d)), compared to predictions from several models. The error bars on all data points (which, in (a) and (c), are smaller than the size of the markers) reflect just statistical uncertainties, with systematic uncertainties plotted as grey bands.

The NLO MC POWHEG matched to the HERWIG parton shower reproduces the dependence on $p_{\mathrm{T}}$, but not the normalisation, which is overestimated by $\sim 40 \%$. CASCADE predicts a different $p_{\mathrm{T}}$-dependence which might come from the initial-state parton showers [55] which are very sensitive to the unintegrated parton densities. The HEJ code, used only at parton level here, describes the data reasonably well.

The discrepancies in the comparison of dijet data with MC models are larger for jets at central values of $\eta$ in figures 8 (a) and (c). In the case of forward jets, the comparison 


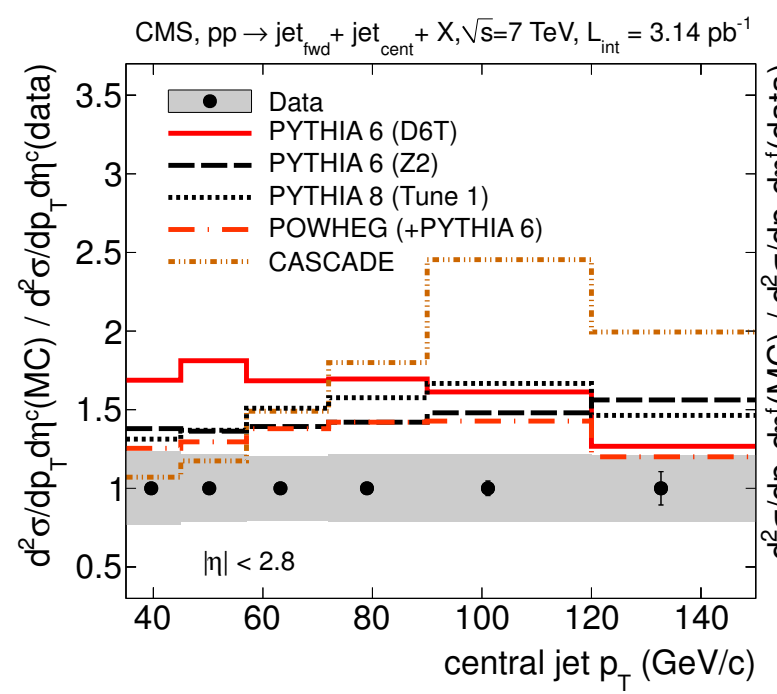

(a)

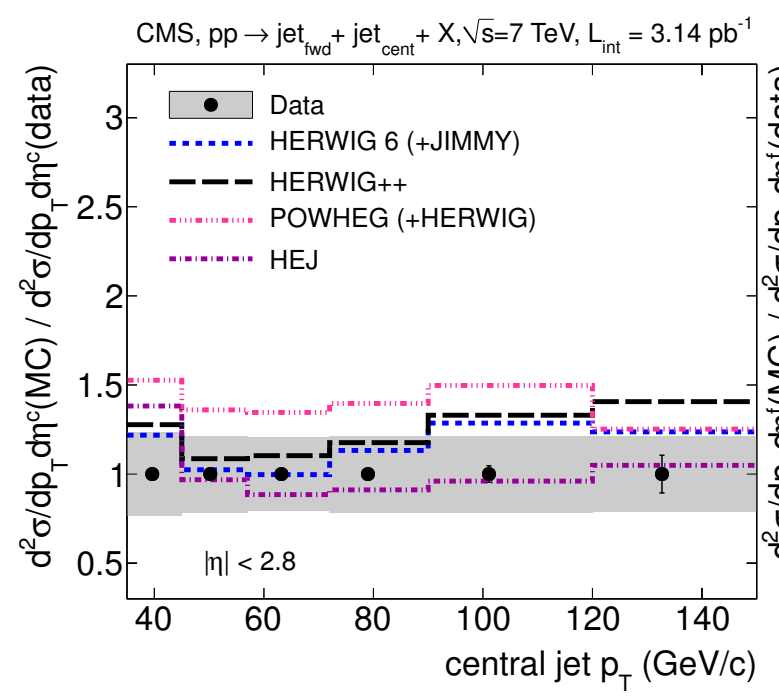

(c)

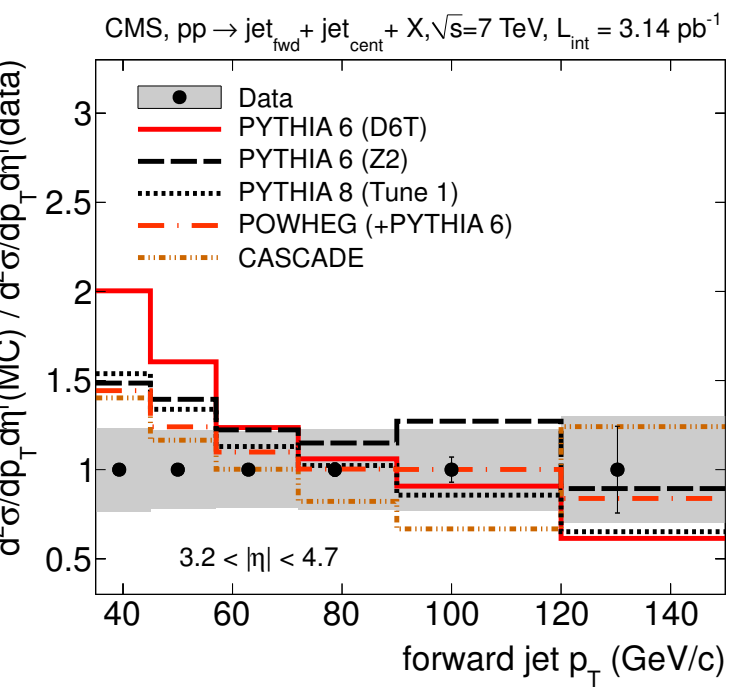

(b)

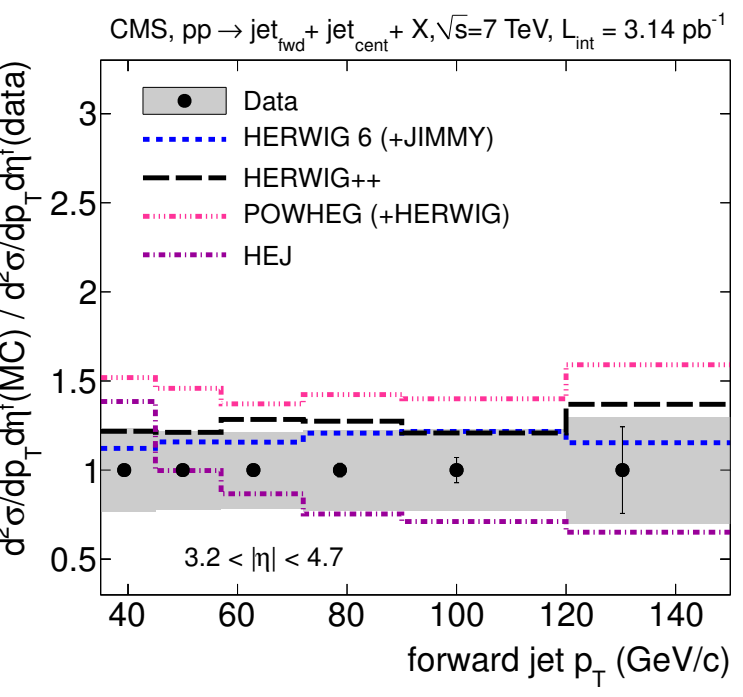

(d)

Figure 8. Ratio of theory to data for differential cross sections as a function of $p_{\mathrm{T}}$, for central ((a) and (c)) and forward ((b) and (d)) jets produced in dijet events. The error bars on all data points reflect just statistical uncertainties, with systematic uncertainties plotted as grey bands.

of the inclusive $p_{\mathrm{T}}$ spectrum (figure 5 ) with that requiring the simultaneous presence of a jet in the central pseudorapidity region (figures 7 (b) and (d)) shows that the inclusive spectrum is about a factor of four higher in the lowest $p_{\mathrm{T}}$ bin but that both distributions agree progressively better at larger $p_{\mathrm{T}}$ values. This suggests that inclusive forward jets of $p_{\mathrm{T}} \approx 35-70 \mathrm{GeV} / c$ may be balanced by other forward jets or by soft central jets that do not surpass the $p_{\mathrm{T}}$ threshold of $35 \mathrm{GeV} / c$, thereby producing the overall deficit of central jets in the data shown in figures 8 (a) and (c). These results confirm that the mechanisms for 
multijet production over large intervals in $\eta$ currently implemented in QCD models used at the LHC can be further constrained through measurements of differential distributions like those presented here. This work complements other studies based on jet multiplicities or on $p_{\mathrm{T}}$-integrated cross sections as a function of the jet $\Delta \eta$ separation.

\section{Summary}

The inclusive production cross section for forward jets has been measured as a function of $p_{\mathrm{T}}$, in the pseudorapidity range $3.2<|\eta|<4.7$. Also, the single-jet cross section has been measured for the two leading jets in inclusive dijet events containing at least one forward and one central jet (defined by the region $|\eta|<2.8$ ). The data are based on $3.14 \mathrm{pb}^{-1}$ of integrated luminosity collected by the CMS detector in proton-proton collisions at $\sqrt{s}=7 \mathrm{TeV}$. Jets were reconstructed using the anti- $k_{T}$ algorithm $(R=0.5)$ in the $p_{\mathrm{T}}$ range $35-150 \mathrm{GeV} / c$. The total systematic uncertainties are $\pm(20-30) \%$, dominated by the absolute jet energy scale. Within the current experimental and theoretical uncertainties, perturbative QCD calculations, as implemented in the parton-shower event generators PYTHIA and HERWIG, as well as in the combined DGLAP+BFKL resummation of the CASCADE model and with the extra wide-angle gluon radiations included in the HEJ model, are in good agreement with the measured inclusive single-jet forward cross section. Calculations at NLO accuracy using recent sets of PDF also describe correctly the $p_{\mathrm{T}}$ dependence of the data, although the predicted absolute cross sections are about $20 \%$ too large.

For the inclusive dijet events, all PYTHIA tunes are found to overestimate the absolute cross sections for the simultaneous production of jets above $p_{\mathrm{T}}=35 \mathrm{GeV} / c$ in the central and forward regions. The agreement is poor for the entire central-jet spectrum and at smallest $p_{\mathrm{T}}$ for forward jets. The HERWIG event generator provides a better description of both differential cross sections, including their normalisations. NLO contributions from POWHEG to both of these parton-shower MC generators enhance the cross sections at all $p_{\mathrm{T}}$ and thereby the disagreement with data. Calculations including resummation of low- $x$ logarithms, as in the CASCADE Monte Carlo, do not reproduce the central-jet spectrum very well, but alternative approaches that account for multijet BFKL-like topologies, such as in the HEJ model, show reasonable agreement with the dijet data. The above measurements provide a valuable test of perturbative QCD in the forward region of proton-proton collisions at the highest available energies, as well as a first check of models for multijet production which are relevant to other processes at the LHC, such as vector-boson fusion, characterised by forward/backward jet production.

\section{Acknowledgments}

We wish to express our gratitude to Jeppe Andersen and Jenni Smillie for fruitful discussions on the comparisons of the measurements with the HEJ model predictions, and to Gavin Salam for useful exchanges on the theoretical calculations.

We wish to congratulate our colleagues in the CERN accelerator departments for the excellent performance of the LHC machine. We thank the technical and administrative 
staff at CERN and other CMS institutes. This work was supported by the Austrian Federal Ministry of Science and Research; the Belgium Fonds de la Recherche Scientifique, and Fonds voor Wetenschappelijk Onderzoek; the Brazilian Funding Agencies (CNPq, CAPES, FAPERJ, and FAPESP); the Bulgarian Ministry of Education and Science; CERN; the Chinese Academy of Sciences, Ministry of Science and Technology, and National Natural Science Foundation of China; the Colombian Funding Agency (COLCIENCIAS); the Croatian Ministry of Science, Education and Sport; the Research Promotion Foundation, Cyprus; the Estonian Academy of Sciences and NICPB; the Academy of Finland, Finnish Ministry of Education and Culture, and Helsinki Institute of Physics; the Institut National de Physique Nucléaire et de Physique des Particules / CNRS, and Commissariat à l'Énergie Atomique et aux Énergies Alternatives / CEA, France; the Bundesministerium für Bildung und Forschung, Deutsche Forschungsgemeinschaft, and Helmholtz-Gemeinschaft Deutscher Forschungszentren, Germany; the General Secretariat for Research and Technology, Greece; the National Scientific Research Foundation, and National Office for Research and Technology, Hungary; the Department of Atomic Energy and the Department of Science and Technology, India; the Institute for Studies in Theoretical Physics and Mathematics, Iran; the Science Foundation, Ireland; the Istituto Nazionale di Fisica Nucleare, Italy; the Korean Ministry of Education, Science and Technology and the World Class University program of NRF, Korea; the Lithuanian Academy of Sciences; the Mexican Funding Agencies (CINVESTAV, CONACYT, SEP, and UASLP-FAI); the Ministry of Science and Innovation, New Zealand; the Pakistan Atomic Energy Commission; the Ministry of Science and Higher Education and the National Science Centre, Poland; the Fundação para a Ciência e a Tecnologia, Portugal; JINR (Armenia, Belarus, Georgia, Ukraine, Uzbekistan); the Ministry of Education and Science of the Russian Federation, the Federal Agency of Atomic Energy of the Russian Federation, Russian Academy of Sciences, and the Russian Foundation for Basic Research; the Ministry of Science and Technological Development of Serbia; the Ministerio de Ciencia e Innovación, and Programa Consolider-Ingenio 2010, Spain; the Swiss Funding Agencies (ETH Board, ETH Zurich, PSI, SNF, UniZH, Canton Zurich, and SER); the National Science Council, Taipei; the Scientific and Technical Research Council of Turkey, and Turkish Atomic Energy Authority; the Science and Technology Facilities Council, U.K.; the US Department of Energy, and the US National Science Foundation.

Individuals have received support from the Marie-Curie programme and the European Research Council (European Union); the Leventis Foundation; the A. P. Sloan Foundation; the Alexander von Humboldt Foundation; the Belgian Federal Science Policy Office; the Fonds pour la Formation à la Recherche dans l'Industrie et dans l'Agriculture (FRIA-Belgium); the Agentschap voor Innovatie door Wetenschap en Technologie (IWTBelgium); the Council of Science and Industrial Research, India; and the HOMING PLUS programme of Foundation for Polish Science, cofinanced from European Union, Regional Development Fund.

Open Access. This article is distributed under the terms of the Creative Commons Attribution License which permits any use, distribution and reproduction in any medium, provided the original author(s) and source are credited. 


\section{References}

[1] S. Ellis, J. Huston, K. Hatakeyama, P. Loch and M. Tonnesmann, Jets in hadron-hadron collisions, Prog. Part. Nucl. Phys. 60 (2008) 484 [arXiv:0712.2447] [InSPIRE].

[2] CMS collaboration, S. Chatrchyan et al., Measurement of the inclusive jet cross section in $p p$ collisions at $\sqrt{s}=7$ TeV, Phys. Rev. Lett. 107 (2011) 132001 [arXiv:1106.0208] [INSPIRE].

[3] ATLAS collaboration, G. Aad et al., Measurement of inclusive jet and dijet production in pp collisions at $\sqrt{s}=7 \mathrm{TeV}$ using the ATLAS detector, arXiv:1112.6297 [INSPIRE].

[4] D0 collaboration, V. Abazov et al., Measurement of the inclusive jet cross-section in $p \bar{p}$ collisions at $\sqrt{s}=1.96$ TeV, Phys. Rev. Lett. 101 (2008) 062001 [arXiv:0802.2400] [INSPIRE].

[5] CDF collaboration, T. Aaltonen et al., Measurement of the inclusive jet cross section at the Fermilab Tevatron p $\bar{p}$ collider using a cone-based jet algorithm, Phys. Rev. D 78 (2008) 052006 [Erratum ibid. D 79 (2009) 119902] [arXiv:0807.2204] [INSPIRE].

[6] H1 and ZEUS collaborations, F. Aaron et al., Combined measurement and QCD analysis of the inclusive $e^{ \pm} p$ scattering cross sections at HERA, JHEP 01 (2010) 109 [arXiv:0911.0884] [INSPIRE].

[7] CMS collaboration, S. Cerci and D. d'Enterria, Low-x QCD studies with forward jets in proton-proton collisions at $\sqrt{s}=14$ TeV, AIP Conf. Proc. 1105 (2009) 28 [arXiv:0812.2665] [INSPIRE].

[8] V. Gribov and L. Lipatov, Deep inelastic ep scattering in perturbation theory, Sov. J. Nucl. Phys. 15 (1972) 438 [Yad. Fiz. 15 (1972) 781] [inSPIRE].

[9] L. Lipatov, The parton model and perturbation theory, Sov. J. Nucl. Phys. 20 (1975) 94 [Yad. Fiz. 20 (1974) 181] [INSPIRE].

[10] G. Altarelli and G. Parisi, Asymptotic freedom in parton language, Nucl. Phys. B 126 (1977) 298 [INSPIRE].

[11] Y.L. Dokshitzer, Calculation of the structure functions for deep inelastic scattering and $e^{+} e^{-}$ annihilation by perturbation theory in quantum chromodynamics, Sov. Phys. JETP 46 (1977) 641 [Zh. Eksp. Teor. Fiz. 73 (1977) 1216] [INSPIRE].

[12] E. Kuraev, L. Lipatov and V.S. Fadin, Multi-Reggeon processes in the Yang-Mills theory, Sov. Phys. JETP 44 (1976) 443 [Zh. Eksp. Teor. Fiz. 71 (1976) 840] [InSPIRE].

[13] E. Kuraev, L. Lipatov and V.S. Fadin, The Pomeranchuk singularity in non-Abelian gauge theories, Sov. Phys. JETP 45 (1977) 199 [Zh. Eksp. Teor. Fiz. 72 (1977) 377] [InSPIRE].

[14] I. Balitsky and L. Lipatov, The Pomeranchuk singularity in quantum chromodynamics, Sov. J. Nucl. Phys. 28 (1978) 822 [Yad. Fiz. 28 (1978) 1597] [InSPIRE].

[15] M. Ciafaloni, Coherence effects in initial jets at small $Q^{2} / s$, Nucl. Phys. B 296 (1988) 49 [INSPIRE].

[16] S. Catani, F. Fiorani and G. Marchesini, QCD coherence in initial state radiation, Phys. Lett. B 234 (1990) 339 [INSPIRE].

[17] S. Catani, F. Fiorani and G. Marchesini, Small $x$ behavior of initial state radiation in perturbative QCD, Nucl. Phys. B 336 (1990) 18 [INSPIRE]. 
[18] G. Marchesini, QCD coherence in the structure function and associated distributions at small x, Nucl. Phys. B 445 (1995) 49 [hep-ph/9412327] [INSPIRE].

[19] F. Gelis et al., The Color Glass Condensate, Ann. Rev. Nucl. Part. Sci. 60 (2010) 463 [arXiv: 1002.0333] [INSPIRE].

[20] T. Figy, C. Oleari and D. Zeppenfeld, Next-to-leading order jet distributions for Higgs boson production via weak boson fusion, Phys. Rev. D 68 (2003) 073005 [hep-ph/0306109] [INSPIRE].

[21] C.F. Berger, C. Marcantonini, I.W. Stewart, F.J. Tackmann and W.J. Waalewijn, Higgs production with a central jet veto at NNLL+NNLO, JHEP 04 (2011) 092 [arXiv:1012.4480] [INSPIRE].

[22] J. Butterworth, B. Cox and J.R. Forshaw, $W W$ scattering at the CERN LHC, Phys. Rev. D 65 (2002) 096014 [hep-ph/0201098] [INSPIRE].

[23] ATLAS collaboration, G. Aad et al., Measurement of dijet production with a veto on additional central jet activity in pp collisions at $\sqrt{s}=7 \mathrm{TeV}$ using the ATLAS detector, JHEP 09 (2011) 053 [arXiv:1107.1641] [INSPIRE].

[24] T. Sjöstrand, S. Mrenna and P.Z. Skands, PYTHIA 6.4 physics and manual, JHEP 05 (2006) 026 [hep-ph/0603175] [INSPIRE].

[25] T. Sjöstrand, S. Mrenna and P.Z. Skands, A brief introduction to PYTHIA 8.1, Comput. Phys. Commun. 178 (2008) 852 [arXiv:0710.3820] [INSPIRE].

[26] G. Marchesini et al., HERWIG: a Monte Carlo event generator for simulating hadron emission reactions with interfering gluons. Version 5.1 - april 1991, Comput. Phys. Commun. 67 (1992) 465 [INSPIRE].

[27] J. Butterworth, J.R. Forshaw and M. Seymour, Multiparton interactions in photoproduction at HERA, Z. Phys. C 72 (1996) 637 [hep-ph/9601371] [InSPIRE].

[28] M. Bahr et al., HERWIG++ 2.2 release note, arXiv:0804.3053 [INSPIRE].

[29] Z. Nagy, Three jet cross-sections in hadron hadron collisions at next-to-leading order, Phys. Rev. Lett. 88 (2002) 122003 [hep-ph/0110315] [INSPIRE].

[30] Z. Nagy, Next-to-leading order calculation of three jet observables in hadron hadron collision, Phys. Rev. D 68 (2003) 094002 [hep-ph/0307268] [INSPIRE].

[31] S. Frixione, P. Nason and C. Oleari, Matching NLO QCD computations with parton shower simulations: the POWHEG method, JHEP 11 (2007) 070 [arXiv: 0709.2092] [INSPIRE].

[32] H. Jung and G. Salam, Hadronic final state predictions from CCFM: the hadron level Monte Carlo generator CASCADE, Eur. Phys. J. C 19 (2001) 351 [hep-ph/0012143] [INSPIRE].

[33] H. Jung et al., The CCFM Monte Carlo generator CASCADE version 2.2.03, Eur. Phys. J. C 70 (2010) 1237 [arXiv: 1008.0152] [INSPIRE].

[34] J.R. Andersen and J.M. Smillie, Constructing all-order corrections to multi-jet rates, JHEP 01 (2010) 039 [arXiv: 0908.2786] [INSPIRE].

[35] J.R. Andersen and J.M. Smillie, Multiple jets at the LHC with high energy jets, JHEP 06 (2011) 010 [arXiv:1101.5394] [INSPIRE].

[36] CMS collaboration, The CMS experiment at the CERN LHC, 2008 JINST 3 S08004 [INSPIRE]. 
[37] CMS collaboration, S. Chatrchyan et al., Performance and operation of the CMS electromagnetic calorimeter, 2010 JINST 5 T03010 [arXiv:0910.3423] [INSPIRE].

[38] CMS collaboration, S. Chatrchyan et al., Performance of the CMS hadron calorimeter with cosmic ray muons and LHC beam data, 2010 JINST 5 T03012 [arXiv:0911.4991] [INSPIRE].

[39] G. Bayatian et al., Design, performance and calibration of the CMS forward calorimeter wedges, Eur. Phys. J. C 53 (2008) 139 [InSPIRE].

[40] CMS collaboration, CMS physics technical design report volume I: detector performance and software, CERN-LHCC-2006-001, section 11.6.3, CERN, Geneva Switzerland (2006) [CMS-TDR-008-1] [INSPIRE].

[41] CMS collaboration, Performance of jet algorithms in CMS, CMS-PAS-JME-07-003, CERN, Geneva Switzerland (2007) [INSPIRE].

[42] CMS collaboration, Tracking and vertexing results from first collisions, CMS-PAS-TRK-10-001, CERN, Geneva Switzerland (2010) [INSPIRE].

[43] M. Cacciari, G.P. Salam and G. Soyez, The anti- $k_{t}$ jet clustering algorithm, JHEP 04 (2008) 063 [arXiv: 0802.1189] [INSPIRE].

[44] M. Cacciari, G.P. Salam and G. Soyez, FastJet user manual, Eur. Phys. J. C 72 (2012) 1896 [arXiv:1111.6097] [INSPIRE].

[45] CMS collaboration, S. Chatrchyan et al., Determination of jet energy calibration and transverse momentum resolution in CMS, 2011 JINST 6 P11002 [arXiv:1107.4277] [INSPIRE].

[46] GEANT4 collaboration, S. Agostinelli et al., GEANT4: a simulation toolkit, Nucl. Instrum. Meth. A 506 (2003) 250 [INSPIRE].

[47] T. Adye, Unfolding algorithms and tests using RooUnfold, arXiv:1105.1160 [INSPIRE].

[48] S. Berman, J. Bjorken and J.B. Kogut, Inclusive processes at high transverse momentum, Phys. Rev. D 4 (1971) 3388 [inSPIRE].

[49] R. Feynman, R. Field and G. Fox, A quantum chromodynamic approach for the large transverse momentum production of particles and jets, Phys. Rev. D 18 (1978) 3320 [INSPIRE].

[50] CMS collaboration, Absolute luminosity normalization, CMS-DP-2011-002, CERN, Geneva Switzerland (2011).

[51] R. Field, Early LHC underlying event data - findings and surprises, arXiv:1010.3558 [INSPIRE].

[52] S. Alioli, K. Hamilton, P. Nason, C. Oleari and E. Re, Jet pair production in POWHEG, JHEP 04 (2011) 081 [arXiv: 1012.3380] [InSPIRE].

[53] T. Kluge, K. Rabbertz and M. Wobisch, FastNLO: fast $p Q C D$ calculations for PDF fits, in $14^{\text {th }}$ International Workshop on Deep Inelastic Scattering (DIS 2006), Tsukuba Japan April 20-24 2006, pg. 483 [hep-ph/0609285] [INSPIRE].

[54] M. Deak, F. Hautmann, H. Jung and K. Kutak, Forward jet production at the Large Hadron Collider, JHEP 09 (2009) 121 [arXiv:0908.0538] [INSPIRE].

[55] M. Deak, F. Hautmann, H. Jung and K. Kutak, Forward-central jet correlations at the Large Hadron Collider, arXiv:1012.6037 [INSPIRE]. 
[56] J. Pumplin et al., New generation of parton distributions with uncertainties from global QCD analysis, JHEP 07 (2002) 012 [hep-ph/0201195] [INSPIRE].

[57] CTEQ collaboration, H. Lai et al., Global QCD analysis of parton structure of the nucleon: CTEQ5 parton distributions, Eur. Phys. J. C 12 (2000) 375 [hep-ph/9903282] [INSPIRE].

[58] A.D. Martin, R. Roberts, W. Stirling and R. Thorne, MRST2001: partons and $\alpha_{s}$ from precise deep inelastic scattering and Tevatron jet data, Eur. Phys. J. C 23 (2002) 73 [hep-ph/0110215] [INSPIRE].

[59] H.-L. Lai et al., New parton distributions for collider physics, Phys. Rev. D 82 (2010) 074024 [arXiv: 1007.2241] [InSPIRE].

[60] P.Z. Skands, The Perugia tunes, arXiv:0905.3418 [inSPIRE].

[61] H. Jung, Un-integrated PDFs in CCFM, hep-ph/0411287 [INSPIRE].

[62] A. Martin, W. Stirling, R. Thorne and G. Watt, Parton distributions for the LHC, Eur. Phys. J. C 63 (2009) 189 [arXiv:0901.0002] [InSPIRE].

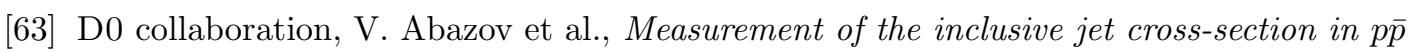
collisions at $\sqrt{s}=1.96 \mathrm{TeV}$, Phys. Rev. Lett. 101 (2008) 062001 [arXiv:0802.2400] [INSPIRE].

[64] S. Alekhin et al., The PDF4LHC working group interim report, arXiv:1101.0536 [INSPIRE].

[65] R.D. Ball et al., Impact of heavy quark masses on parton distributions and LHC phenomenology, Nucl. Phys. B 849 (2011) 296 [arXiv:1101.1300] [INSPIRE].

[66] A. Banfi, G.P. Salam and G. Zanderighi, Phenomenology of event shapes at hadron colliders, JHEP 06 (2010) 038 [arXiv: 1001.4082] [InSPIRE].

[67] Particle Data Group collaboration, Review of particle physics, Phys. Lett. B 667 (2008) 101 [INSPIRE].

[68] G. Lafferty and T. Wyatt, Where to stick your data points: the treatment of measurements within wide bins, Nucl. Instrum. Meth. A 355 (1995) 541 [INSPIRE].

[69] CMS collaboration, Comparison of inclusive jet and dijet mass cross sections at $\sqrt{s}=7 \mathrm{TeV}$ with predictions of perturbative QCD, CMS-NOTE-2011-004, CERN, Geneva Switzerland (2011). 


\section{The CMS collaboration}

\section{Yerevan Physics Institute, Yerevan, Armenia}

S. Chatrchyan, V. Khachatryan, A.M. Sirunyan, A. Tumasyan

Institut für Hochenergiephysik der OeAW, Wien, Austria

W. Adam, T. Bergauer, M. Dragicevic, J. Erö, C. Fabjan, M. Friedl, R. Frühwirth, V.M. Ghete, J. Hammer ${ }^{1}$, M. Hoch, N. Hörmann, J. Hrubec, M. Jeitler, W. Kiesenhofer, A. Knapitsch, M. Krammer, D. Liko, I. Mikulec, M. Pernicka ${ }^{\dagger}$, B. Rahbaran, C. Rohringer, H. Rohringer, R. Schöfbeck, J. Strauss, A. Taurok, F. Teischinger, P. Wagner, W. Waltenberger, G. Walzel, E. Widl, C.-E. Wulz

National Centre for Particle and High Energy Physics, Minsk, Belarus

V. Mossolov, N. Shumeiko, J. Suarez Gonzalez

Universiteit Antwerpen, Antwerpen, Belgium

S. Bansal, L. Benucci, E.A. De Wolf, X. Janssen, S. Luyckx, T. Maes, L. Mucibello,

S. Ochesanu, B. Roland, R. Rougny, M. Selvaggi, H. Van Haevermaet, P. Van Mechelen, N. Van Remortel, A. Van Spilbeeck

Vrije Universiteit Brussel, Brussel, Belgium

F. Blekman, S. Blyweert, J. D'Hondt, R. Gonzalez Suarez, A. Kalogeropoulos, M. Maes, A. Olbrechts, W. Van Doninck, P. Van Mulders, G.P. Van Onsem, I. Villella

Université Libre de Bruxelles, Bruxelles, Belgium

O. Charaf, B. Clerbaux, G. De Lentdecker, V. Dero, A.P.R. Gay, G.H. Hammad, T. Hreus, A. Léonard, P.E. Marage, L. Thomas, C. Vander Velde, P. Vanlaer, J. Wickens

\section{Ghent University, Ghent, Belgium}

V. Adler, K. Beernaert, A. Cimmino, S. Costantini, M. Grunewald, B. Klein, J. Lellouch, A. Marinov, J. Mccartin, A.A. Ocampo Rios, D. Ryckbosch, N. Strobbe, F. Thyssen, M. Tytgat, L. Vanelderen, P. Verwilligen, S. Walsh, N. Zaganidis

Université Catholique de Louvain, Louvain-la-Neuve, Belgium

S. Basegmez, G. Bruno, J. Caudron, L. Ceard, J. De Favereau De Jeneret, C. Delaere, D. Favart, L. Forthomme, A. Giammanco ${ }^{2}$, G. Grégoire, J. Hollar, V. Lemaitre, J. Liao, O. Militaru, C. Nuttens, D. Pagano, A. Pin, K. Piotrzkowski, N. Schul

Université de Mons, Mons, Belgium

N. Beliy, T. Caebergs, E. Daubie

Centro Brasileiro de Pesquisas Fisicas, Rio de Janeiro, Brazil

G.A. Alves, D. De Jesus Damiao, M.E. Pol, M.H.G. Souza

Universidade do Estado do Rio de Janeiro, Rio de Janeiro, Brazil

W.L. Aldá Júnior, W. Carvalho, A. Custódio, E.M. Da Costa, C. De Oliveira Martins, S. Fonseca De Souza, D. Matos Figueiredo, L. Mundim, H. Nogima, V. Oguri, W.L. Prado Da Silva, A. Santoro, S.M. Silva Do Amaral, A. Sznajder 
Instituto de Fisica Teorica, Universidade Estadual Paulista, Sao Paulo, Brazil T.S. Anjos $^{3}$, C.A. Bernardes ${ }^{3}$, F.A. Dias ${ }^{4}$, T.R. Fernandez Perez Tomei, E. M. Gregores ${ }^{3}$, C. Lagana, F. Marinho, P.G. Mercadante ${ }^{3}$, S.F. Novaes, Sandra S. Padula

Institute for Nuclear Research and Nuclear Energy, Sofia, Bulgaria

N. Darmenov ${ }^{1}$, V. Genchev ${ }^{1}$, P. Iaydjiev ${ }^{1}$, S. Piperov, M. Rodozov, S. Stoykova, G. Sultanov, V. Tcholakov, R. Trayanov, M. Vutova

University of Sofia, Sofia, Bulgaria

A. Dimitrov, R. Hadjiiska, A. Karadzhinova, V. Kozhuharov, L. Litov, B. Pavlov, P. Petkov

Institute of High Energy Physics, Beijing, China

J.G. Bian, G.M. Chen, H.S. Chen, C.H. Jiang, D. Liang, S. Liang, X. Meng, J. Tao, J. Wang, J. Wang, X. Wang, Z. Wang, H. Xiao, M. Xu, J. Zang, Z. Zhang

State Key Lab. of Nucl. Phys. and Tech., Peking University, Beijing, China Y. Ban, S. Guo, Y. Guo, W. Li, S. Liu, Y. Mao, S.J. Qian, H. Teng, S. Wang, B. Zhu, W. Zou

Universidad de Los Andes, Bogota, Colombia

A. Cabrera, B. Gomez Moreno, A.F. Osorio Oliveros, J.C. Sanabria

Technical University of Split, Split, Croatia

N. Godinovic, D. Lelas, R. Plestina ${ }^{5}$, D. Polic, I. Puljak ${ }^{1}$

University of Split, Split, Croatia

Z. Antunovic, M. Dzelalija, M. Kovac

Institute Rudjer Boskovic, Zagreb, Croatia

V. Brigljevic, S. Duric, K. Kadija, J. Luetic, S. Morovic

University of Cyprus, Nicosia, Cyprus

A. Attikis, M. Galanti, J. Mousa, C. Nicolaou, F. Ptochos, P.A. Razis

Charles University, Prague, Czech Republic

M. Finger, M. Finger Jr.

Academy of Scientific Research and Technology of the Arab Republic of Egypt, Egyptian Network of High Energy Physics, Cairo, Egypt

Y. Assran ${ }^{6}$, A. Ellithi Kamel ${ }^{7}$, S. Khalil ${ }^{8}$, M.A. Mahmoud ${ }^{9}$, A. Radi ${ }^{10}$

National Institute of Chemical Physics and Biophysics, Tallinn, Estonia

A. Hektor, M. Kadastik, M. Müntel, M. Raidal, L. Rebane, A. Tiko

Department of Physics, University of Helsinki, Helsinki, Finland

V. Azzolini, P. Eerola, G. Fedi, M. Voutilainen

Helsinki Institute of Physics, Helsinki, Finland

S. Czellar, J. Härkönen, A. Heikkinen, V. Karimäki, R. Kinnunen, M.J. Kortelainen,

T. Lampén, K. Lassila-Perini, S. Lehti, T. Lindén, P. Luukka, T. Mäenpää, E. Tuominen,

J. Tuominiemi, E. Tuovinen, D. Ungaro, L. Wendland 
Lappeenranta University of Technology, Lappeenranta, Finland

K. Banzuzi, A. Korpela, T. Tuuva

Laboratoire d'Annecy-le-Vieux de Physique des Particules, IN2P3-CNRS, Annecy-le-Vieux, France

D. Sillou

\section{DSM/IRFU, CEA/Saclay, Gif-sur-Yvette, France}

M. Besancon, S. Choudhury, M. Dejardin, D. Denegri, B. Fabbro, J.L. Faure, F. Ferri, S. Ganjour, A. Givernaud, P. Gras, G. Hamel de Monchenault, P. Jarry, E. Locci, J. Malcles, M. Marionneau, L. Millischer, J. Rander, A. Rosowsky, I. Shreyber, M. Titov

Laboratoire Leprince-Ringuet, Ecole Polytechnique, IN2P3-CNRS, Palaiseau, France

S. Baffioni, F. Beaudette, L. Benhabib, L. Bianchini, M. Bluj ${ }^{11}$, C. Broutin, P. Busson, C. Charlot, N. Daci, T. Dahms, L. Dobrzynski, S. Elgammal, R. Granier de Cassagnac, M. Haguenauer, P. Miné, C. Mironov, C. Ochando, P. Paganini, D. Sabes, R. Salerno, Y. Sirois, C. Thiebaux, C. Veelken, A. Zabi

Institut Pluridisciplinaire Hubert Curien, Université de Strasbourg, Université de Haute Alsace Mulhouse, CNRS/IN2P3, Strasbourg, France

J.-L. Agram ${ }^{12}$, J. Andrea, D. Bloch, D. Bodin, J.-M. Brom, M. Cardaci, E.C. Chabert, C. Collard, E. Conte ${ }^{12}$, F. Drouhin ${ }^{12}$, C. Ferro, J.-C. Fontaine ${ }^{12}$, D. Gelé, U. Goerlach, S. Greder, P. Juillot, M. Karim ${ }^{12}$, A.-C. Le Bihan, P. Van Hove

Centre de Calcul de l'Institut National de Physique Nucleaire et de Physique des Particules (IN2P3), Villeurbanne, France

F. Fassi, D. Mercier

Université de Lyon, Université Claude Bernard Lyon 1, CNRS-IN2P3, Institut de Physique Nucléaire de Lyon, Villeurbanne, France

C. Baty, S. Beauceron, N. Beaupere, M. Bedjidian, O. Bondu, G. Boudoul, D. Boumediene, H. Brun, J. Chasserat, R. Chierici ${ }^{1}$, D. Contardo, P. Depasse, H. El Mamouni, A. Falkiewicz, J. Fay, S. Gascon, M. Gouzevitch, B. Ille, T. Kurca, T. Le Grand, M. Lethuillier, L. Mirabito, S. Perries, V. Sordini, S. Tosi, Y. Tschudi, P. Verdier, S. Viret

Institute of High Energy Physics and Informatization, Tbilisi State University, Tbilisi, Georgia

D. Lomidze

RWTH Aachen University, I. Physikalisches Institut, Aachen, Germany

G. Anagnostou, S. Beranek, M. Edelhoff, L. Feld, N. Heracleous, O. Hindrichs, R. Jussen, K. Klein, J. Merz, A. Ostapchuk, A. Perieanu, F. Raupach, J. Sammet, S. Schael, D. Sprenger, H. Weber, B. Wittmer, V. Zhukov ${ }^{13}$

RWTH Aachen University, III. Physikalisches Institut A, Aachen, Germany

M. Ata, E. Dietz-Laursonn, M. Erdmann, T. Hebbeker, C. Heidemann, K. Hoepfner, T. Klimkovich, D. Klingebiel, P. Kreuzer, D. Lanske ${ }^{\dagger}$, J. Lingemann, C. Magass, M. Mer- 
schmeyer, A. Meyer, P. Papacz, H. Pieta, H. Reithler, S.A. Schmitz, L. Sonnenschein, J. Steggemann, D. Teyssier, M. Weber

\section{RWTH Aachen University, III. Physikalisches Institut B, Aachen, Germany}

M. Bontenackels, V. Cherepanov, M. Davids, G. Flügge, H. Geenen, M. Geisler, W. Haj Ahmad, F. Hoehle, B. Kargoll, T. Kress, Y. Kuessel, A. Linn, A. Nowack, L. Perchalla, O. Pooth, J. Rennefeld, P. Sauerland, A. Stahl, D. Tornier, M.H. Zoeller

\section{Deutsches Elektronen-Synchrotron, Hamburg, Germany}

M. Aldaya Martin, W. Behrenhoff, U. Behrens, M. Bergholz ${ }^{14}$, A. Bethani, K. Borras, A. Cakir, A. Campbell, E. Castro, D. Dammann, G. Eckerlin, D. Eckstein, A. Flossdorf, G. Flucke, A. Geiser, J. Hauk, H. Jung ${ }^{1}$, M. Kasemann, P. Katsas, C. Kleinwort, H. Kluge, A. Knutsson, M. Krämer, D. Krücker, E. Kuznetsova, W. Lange, W. Lohmann ${ }^{14}$, B. Lutz, R. Mankel, I. Marfin, M. Marienfeld, I.-A. Melzer-Pellmann, A.B. Meyer, J. Mnich, A. Mussgiller, S. Naumann-Emme, J. Olzem, A. Petrukhin, D. Pitzl, A. Raspereza, P.M. Ribeiro Cipriano, M. Rosin, J. Salfeld-Nebgen, R. Schmidt ${ }^{14}$, T. Schoerner-Sadenius, N. Sen, A. Spiridonov, M. Stein, J. Tomaszewska, R. Walsh, C. Wissing

\section{University of Hamburg, Hamburg, Germany}

C. Autermann, V. Blobel, S. Bobrovskyi, J. Draeger, H. Enderle, U. Gebbert, M. Görner, T. Hermanns, K. Kaschube, G. Kaussen, H. Kirschenmann, R. Klanner, J. Lange, B. Mura, F. Nowak, N. Pietsch, C. Sander, H. Schettler, P. Schleper, E. Schlieckau, M. Schröder, T. Schum, H. Stadie, G. Steinbrück, J. Thomsen

\section{Institut für Experimentelle Kernphysik, Karlsruhe, Germany}

C. Barth, J. Berger, T. Chwalek, W. De Boer, A. Dierlamm, G. Dirkes, M. Feindt, J. Gruschke, M. Guthoff ${ }^{1}$, C. Hackstein, F. Hartmann, M. Heinrich, H. Held, K.H. Hoffmann, S. Honc, I. Katkov ${ }^{13}$, J.R. Komaragiri, T. Kuhr, D. Martschei, S. Mueller, Th. Müller, M. Niegel, O. Oberst, A. Oehler, J. Ott, T. Peiffer, G. Quast, K. Rabbertz, F. Ratnikov, N. Ratnikova, M. Renz, S. Röcker, C. Saout, A. Scheurer, P. Schieferdecker, F.-P. Schilling, M. Schmanau, G. Schott, H.J. Simonis, F.M. Stober, D. Troendle, J. Wagner-Kuhr, T. Weiler, M. Zeise, E.B. Ziebarth

\section{Institute of Nuclear Physics "Demokritos", Aghia Paraskevi, Greece}

G. Daskalakis, T. Geralis, S. Kesisoglou, A. Kyriakis, D. Loukas, I. Manolakos, A. Markou, C. Markou, C. Mavrommatis, E. Ntomari, E. Petrakou

\section{University of Athens, Athens, Greece}

L. Gouskos, T.J. Mertzimekis, A. Panagiotou, N. Saoulidou, E. Stiliaris

\section{University of Ioánnina, Ioánnina, Greece}

I. Evangelou, C. Foudas ${ }^{1}$, P. Kokkas, N. Manthos, I. Papadopoulos, V. Patras, F.A. Triantis

KFKI Research Institute for Particle and Nuclear Physics, Budapest, Hungary

A. Aranyi, G. Bencze, L. Boldizsar, C. Hajdu ${ }^{1}$, P. Hidas, D. Horvath ${ }^{15}$, A. Kapusi, K. Krajczar ${ }^{16}$, F. Sikler ${ }^{1}$, G. Vesztergombi ${ }^{16}$ 
Institute of Nuclear Research ATOMKI, Debrecen, Hungary

N. Beni, J. Molnar, J. Palinkas, Z. Szillasi, V. Veszpremi

University of Debrecen, Debrecen, Hungary

J. Karancsi, P. Raics, Z.L. Trocsanyi, B. Ujvari

Panjab University, Chandigarh, India

S.B. Beri, V. Bhatnagar, N. Dhingra, R. Gupta, M. Jindal, M. Kaur, J.M. Kohli, M.Z. Mehta, N. Nishu, L.K. Saini, A. Sharma, A.P. Singh, J. Singh, S.P. Singh

University of Delhi, Delhi, India

S. Ahuja, B.C. Choudhary, A. Kumar, A. Kumar, S. Malhotra, M. Naimuddin, K. Ranjan, V. Sharma, R.K. Shivpuri

Saha Institute of Nuclear Physics, Kolkata, India

S. Banerjee, S. Bhattacharya, S. Dutta, B. Gomber, S. Jain, S. Jain, R. Khurana, S. Sarkar

Bhabha Atomic Research Centre, Mumbai, India

R.K. Choudhury, D. Dutta, S. Kailas, V. Kumar, A.K. Mohanty ${ }^{1}$, L.M. Pant, P. Shukla

Tata Institute of Fundamental Research - EHEP, Mumbai, India

T. Aziz, S. Ganguly, M. Guchait ${ }^{17}$, A. Gurtu ${ }^{18}$, M. Maity ${ }^{19}$, D. Majumder, G. Majumder, K. Mazumdar, G.B. Mohanty, B. Parida, A. Saha, K. Sudhakar, N. Wickramage

Tata Institute of Fundamental Research - HECR, Mumbai, India

S. Banerjee, S. Dugad, N.K. Mondal

Institute for Research in Fundamental Sciences (IPM), Tehran, Iran H. Arfaei, H. Bakhshiansohi ${ }^{20}$, S.M. Etesami ${ }^{21}$, A. Fahim ${ }^{20}$, M. Hashemi, H. Hesari, A. Jafari ${ }^{20}$, M. Khakzad, A. Mohammadi ${ }^{22}$, M. Mohammadi Najafabadi, S. Paktinat Mehdiabadi, B. Safarzadeh ${ }^{23}$, M. Zeinali ${ }^{21}$

INFN Sezione di Bari ${ }^{a}$, Università di Bari ${ }^{b}$, Politecnico di Bari ${ }^{c}$, Bari, Italy M. Abbrescia ${ }^{a, b}$, L. Barbone ${ }^{a, b}$, C. Calabria ${ }^{a, b}$, A. Colaleo ${ }^{a}$, D. Creanza ${ }^{a, c}$, N. De

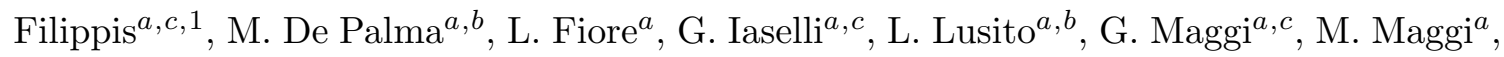
N. Manna ${ }^{a, b}$, B. Marangelli ${ }^{a, b}$, S. My ${ }^{a, c}$, S. Nuzzo ${ }^{a, b}$, N. Pacifico ${ }^{a, b}$, A. Pompili ${ }^{a, b}$, G. Pugliese ${ }^{a, c}$, F. Romano ${ }^{a, c}$, G. Selvaggi ${ }^{a, b}$, L. Silvestris ${ }^{a}$, S. Tupputi ${ }^{a, b}$, G. Zito ${ }^{a}$

INFN Sezione di Bologna ${ }^{a}$, Università di Bologna ${ }^{b}$, Bologna, Italy

G. Abbiendi ${ }^{a}$, A.C. Benvenuti ${ }^{a}$, D. Bonacorsi ${ }^{a}$, S. Braibant-Giacomelli ${ }^{a}, b$, L. Brigliadori ${ }^{a}$, P. Capiluppi ${ }^{a, b}$, A. Castro ${ }^{a, b}$, F.R. Cavallo ${ }^{a}$, M. Cuffiani ${ }^{a, b}$, G.M. Dallavalle ${ }^{a}$, F. Fabbri ${ }^{a}$, A. Fanfani ${ }^{a, b}$, D. Fasanella ${ }^{a, 1}$, P. Giacomelli ${ }^{a}$, C. Grandi ${ }^{a}$, S. Marcellini ${ }^{a}$, G. Masetti ${ }^{a}$, M. Meneghelli ${ }^{a, b}$, A. Montanari ${ }^{a}$, F.L. Navarria ${ }^{a}, b$, F. Odorici ${ }^{a}$, A. Perrotta $^{a}$, F. Primavera ${ }^{a}$, A.M. Rossi ${ }^{a, b}$, T. Rovelli ${ }^{a, b}$, G. Siroli ${ }^{a, b}$, R. Travaglini ${ }^{a, b}$

INFN Sezione di Catania ${ }^{a}$, Università di Catania ${ }^{b}$, Catania, Italy

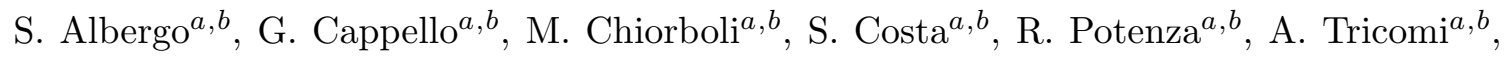
C. Tuve $\mathrm{e}^{a, b}$ 
INFN Sezione di Firenze ${ }^{a}$, Università di Firenze ${ }^{b}$, Firenze, Italy

G. Barbagli ${ }^{a}$, V. Ciulli ${ }^{a, b}$, C. Civinini ${ }^{a}$, R. D’Alessandro ${ }^{a, b}$, E. Focardi ${ }^{a, b}$, S. Frosali ${ }^{a, b}$,

E. Gallo ${ }^{a}$, S. Gonzi ${ }^{a}, b$, M. Meschini $^{a}$, S. Paoletti ${ }^{a}$, G. Sguazzoni ${ }^{a}$, A. Tropiano ${ }^{a, 1}$

INFN Laboratori Nazionali di Frascati, Frascati, Italy

L. Benussi, S. Bianco, S. Colafranceschi ${ }^{24}$, F. Fabbri, D. Piccolo

INFN Sezione di Genova, Genova, Italy

P. Fabbricatore, R. Musenich

INFN Sezione di Milano-Bicocca ${ }^{a}$, Università di Milano-Bicocca ${ }^{b}$, Milano, Italy

A. Benaglia ${ }^{a, b, 1}$, F. De Guio ${ }^{a, b}$, L. Di Matteo ${ }^{a, b}$, S. Gennai $^{a, 1}$, A. Ghezzi $^{a, b}$, S. Malvezzi $^{a}$,

A. Martelli ${ }^{a, b}$, A. Massironi ${ }^{a, b, 1}$, D. Menasce ${ }^{a}$, L. Moroni $^{a}$, M. Paganoni $^{a, b}$, D. Pedrini $^{a}$,

S. Ragazzi ${ }^{a, b}$, N. Redaelli ${ }^{a}$, S. Sala ${ }^{a}$, T. Tabarelli de Fatis ${ }^{a, b}$

INFN Sezione di Napoli ${ }^{a}$, Università di Napoli "Federico II" ${ }^{b}$, Napoli, Italy S. Buontempo ${ }^{a}$, C.A. Carrillo Montoya ${ }^{a, 1}$, N. Cavallo ${ }^{a, 25}$, A. De Cosa $^{a, b}$, O. Dogangun $^{a, b}$,

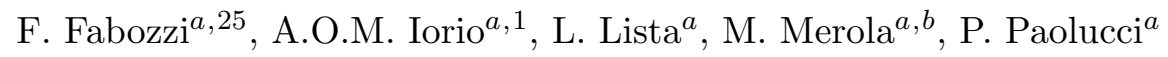

INFN Sezione di Padova ${ }^{a}$, Università di Padova ${ }^{b}$, Università di Trento (Trento) ${ }^{c}$, Padova, Italy

P. Azzi ${ }^{a}$, N. Bacchetta ${ }^{a, 1}$, P. Bellan ${ }^{a, b}$, D. Bisello ${ }^{a, b}$, A. Branca $^{a}$, R. Carlin $^{a, b}$, P. Checchia $^{a}$,

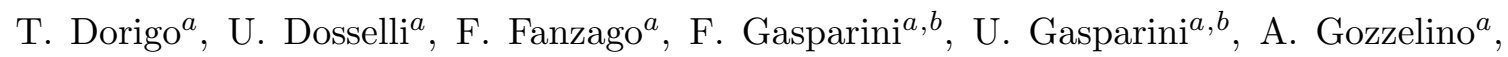

S. Lacaprara ${ }^{a, 26}$, I. Lazzizzera ${ }^{a, c}$, M. Margoni ${ }^{a, b}$, M. Mazzucato $^{a}$, A.T. Meneguzzo ${ }^{a, b}$,

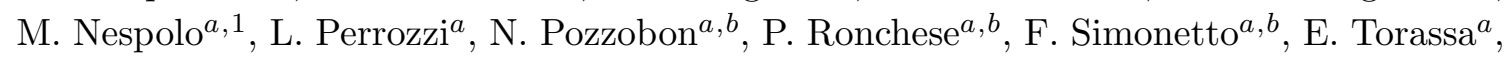

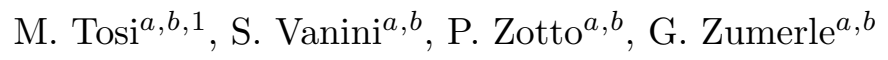

INFN Sezione di Pavia ${ }^{a}$, Università di Pavia ${ }^{b}$, Pavia, Italy

P. Baesso ${ }^{a, b}$, U. Berzano ${ }^{a}$, S.P. Ratti ${ }^{a, b}$, C. Riccardi ${ }^{a, b}$, P. Torre ${ }^{a, b}$, P. Vitulo ${ }^{a, b}$, C. Viviani ${ }^{a, b}$

INFN Sezione di Perugia ${ }^{a}$, Università di Perugia ${ }^{b}$, Perugia, Italy

M. Biasini ${ }^{a, b}$, G.M. Bilei ${ }^{a}$, B. Caponeri ${ }^{a, b}$, L. Fanò ${ }^{a, b}$, P. Lariccia ${ }^{a, b}$, A. Lucaroni ${ }^{a, b, 1}$, G. Mantovani ${ }^{a, b}$, M. Menichelli ${ }^{a}$, A. Nappi $^{a, b}$, F. Romeo $^{a, b}$, A. Santocchia $^{a, b}$, S. Taroni $^{a, b, 1}$, M. Valdata ${ }^{a, b}$

INFN Sezione di Pisa ${ }^{a}$, Università di Pisa ${ }^{b}$, Scuola Normale Superiore di Pisa ${ }^{c}$, Pisa, Italy

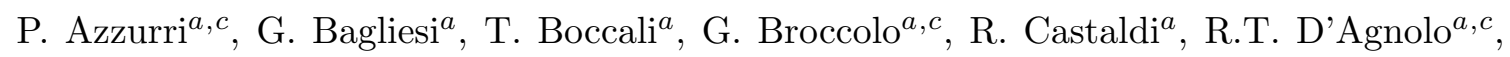

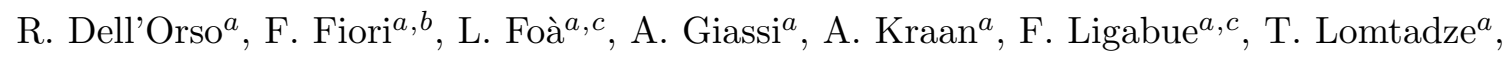

L. Martini ${ }^{a, 27}$, A. Messineo ${ }^{a, b}$, F. Palla ${ }^{a}$, F. Palmonari ${ }^{a}$, A. Rizzi, G. Segneri ${ }^{a}$,

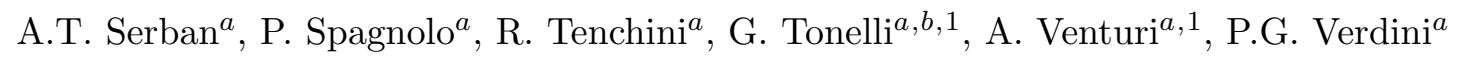

INFN Sezione di Roma ${ }^{a}$, Università di Roma "La Sapienza" ${ }^{b}$, Roma, Italy

L. Barone $e^{a, b}$, F. Cavallari ${ }^{a}$, D. Del Re $e^{a, b, 1}$, M. Diemoz ${ }^{a}$, D. Franci ${ }^{a, b}$, M. Grassi ${ }^{a, 1}$,

E. Longo ${ }^{a, b}$, P. Meridiani ${ }^{a}$, S. Nourbakhsh ${ }^{a}$, G. Organtini ${ }^{a, b}$, F. Pandolfi ${ }^{a}, b$, R. Paramatti ${ }^{a}$,

S. Rahatlou ${ }^{a, b}$, M. Sigamani ${ }^{a}$ 
INFN Sezione di Torino ${ }^{a}$, Università di Torino ${ }^{b}$, Università del Piemonte Orientale (Novara) ${ }^{c}$, Torino, Italy

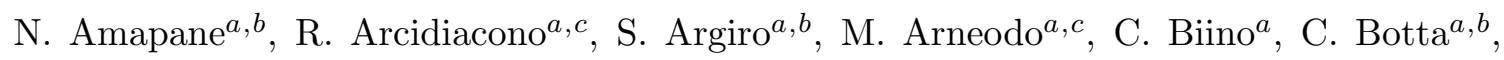
N. Cartiglia ${ }^{a}$, R. Castello ${ }^{a, b}$, M. Costa ${ }^{a, b}$, N. Demaria ${ }^{a}$, A. Graziano ${ }^{a, b}$, C. Mariotti ${ }^{a, 1}$,

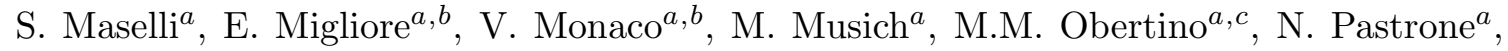

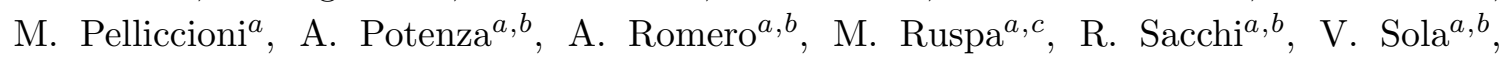
A. Solano ${ }^{a, b}$, A. Staiano ${ }^{a}$, A. Vilela Pereira ${ }^{a}$

INFN Sezione di Trieste ${ }^{a}$, Università di Trieste ${ }^{b}$, Trieste, Italy

S. Belforte ${ }^{a}$, F. Cossutti $^{a}$, G. Della Ricca ${ }^{a, b}$, B. Gobbo ${ }^{a}$, M. Marone $^{a, b}$, D. Montanino $^{a, b, 1}$, A. Penzo ${ }^{a}$

Kangwon National University, Chunchon, Korea

S.G. Heo, S.K. Nam

Kyungpook National University, Daegu, Korea

S. Chang, J. Chung, D.H. Kim, G.N. Kim, J.E. Kim, D.J. Kong, H. Park, S.R. Ro, D.C. Son, T. Son

Chonnam National University, Institute for Universe and Elementary Particles, Kwangju, Korea

J.Y. Kim, Zero J. Kim, S. Song

Konkuk University, Seoul, Korea

H.Y. Jo

Korea University, Seoul, Korea

S. Choi, D. Gyun, B. Hong, M. Jo, H. Kim, T.J. Kim, K.S. Lee, D.H. Moon, S.K. Park, E. Seo, K.S. Sim

University of Seoul, Seoul, Korea

M. Choi, S. Kang, H. Kim, J.H. Kim, C. Park, I.C. Park, S. Park, G. Ryu

Sungkyunkwan University, Suwon, Korea

Y. Cho, Y. Choi, Y.K. Choi, J. Goh, M.S. Kim, B. Lee, J. Lee, S. Lee, H. Seo, I. Yu

Vilnius University, Vilnius, Lithuania

M.J. Bilinskas, I. Grigelionis, M. Janulis, D. Martisiute, P. Petrov, M. Polujanskas, T. Sabonis

Centro de Investigacion y de Estudios Avanzados del IPN, Mexico City, Mexico

H. Castilla-Valdez, E. De La Cruz-Burelo, I. Heredia-de La Cruz, R. Lopez-Fernandez, R. Magaña Villalba, J. Martínez-Ortega, A. Sánchez-Hernández, L.M. Villasenor-Cendejas

Universidad Iberoamericana, Mexico City, Mexico

S. Carrillo Moreno, F. Vazquez Valencia

Benemerita Universidad Autonoma de Puebla, Puebla, Mexico H.A. Salazar Ibarguen 
Universidad Autónoma de San Luis Potosí, San Luis Potosí, Mexico

E. Casimiro Linares, A. Morelos Pineda, M.A. Reyes-Santos

University of Auckland, Auckland, New Zealand

D. Krofcheck

University of Canterbury, Christchurch, New Zealand

A.J. Bell, P.H. Butler, R. Doesburg, S. Reucroft, H. Silverwood

National Centre for Physics, Quaid-I-Azam University, Islamabad, Pakistan

M. Ahmad, M.I. Asghar, H.R. Hoorani, S. Khalid, W.A. Khan, T. Khurshid, S. Qazi, M.A. Shah, M. Shoaib

Institute of Experimental Physics, Faculty of Physics, University of Warsaw, Warsaw, Poland

G. Brona, M. Cwiok, W. Dominik, K. Doroba, A. Kalinowski, M. Konecki, J. Krolikowski

Soltan Institute for Nuclear Studies, Warsaw, Poland

H. Bialkowska, B. Boimska, T. Frueboes, R. Gokieli, M. Górski, M. Kazana, K. Nawrocki, K. Romanowska-Rybinska, M. Szleper, G. Wrochna, P. Zalewski

Laboratório de Instrumentação e Física Experimental de Partículas, Lisboa, Portugal

N. Almeida, P. Bargassa, A. David, P. Faccioli, P.G. Ferreira Parracho, M. Gallinaro, P. Musella, A. Nayak, J. Pela ${ }^{1}$, P.Q. Ribeiro, J. Seixas, J. Varela

Joint Institute for Nuclear Research, Dubna, Russia

S. Afanasiev, I. Belotelov, P. Bunin, M. Gavrilenko, I. Golutvin, I. Gorbunov, A. Kamenev, V. Karjavin, G. Kozlov, A. Lanev, P. Moisenz, V. Palichik, V. Perelygin, S. Shmatov, V. Smirnov, A. Volodko, A. Zarubin

Petersburg Nuclear Physics Institute, Gatchina (St Petersburg), Russia

S. Evstyukhin, V. Golovtsov, Y. Ivanov, V. Kim, P. Levchenko, V. Murzin, V. Oreshkin, I. Smirnov, V. Sulimov, L. Uvarov, S. Vavilov, A. Vorobyev, An. Vorobyev

Institute for Nuclear Research, Moscow, Russia

Yu. Andreev, A. Dermenev, S. Gninenko, N. Golubev, M. Kirsanov, N. Krasnikov, V. Matveev, A. Pashenkov, A. Toropin, S. Troitsky

Institute for Theoretical and Experimental Physics, Moscow, Russia

V. Epshteyn, M. Erofeeva, V. Gavrilov, M. Kossov' ${ }^{1}$, A. Krokhotin, N. Lychkovskaya, V. Popov, G. Safronov, S. Semenov, V. Stolin, E. Vlasov, A. Zhokin

Moscow State University, Moscow, Russia

A. Belyaev, E. Boos, M. Dubinin ${ }^{4}$, A. Ershov, A. Gribushin, L. Khein, O. Kodolova, I. Lokhtin, A. Markina, S. Obraztsov, M. Perfilov, S. Petrushanko, A. Proskuryakov, L. Sarycheva, V. Savrin 
P.N. Lebedev Physical Institute, Moscow, Russia

V. Andreev, M. Azarkin, I. Dremin, M. Kirakosyan, A. Leonidov, G. Mesyats, S.V. Rusakov, A. Vinogradov

State Research Center of Russian Federation, Institute for High Energy Physics, Protvino, Russia

I. Azhgirey, I. Bayshev, S. Bitioukov, V. Grishin ${ }^{1}$, V. Kachanov, D. Konstantinov, A. Korablev, V. Krychkine, V. Petrov, R. Ryutin, A. Sobol, L. Tourtchanovitch, S. Troshin, N. Tyurin, A. Uzunian, A. Volkov

University of Belgrade, Faculty of Physics and Vinca Institute of Nuclear Sciences, Belgrade, Serbia

P. Adzic ${ }^{28}$, M. Djordjevic, M. Ekmedzic, D. Krpic ${ }^{28}$, J. Milosevic

Centro de Investigaciones Energéticas Medioambientales y Tecnológicas (CIEMAT), Madrid, Spain

M. Aguilar-Benitez, J. Alcaraz Maestre, P. Arce, C. Battilana, E. Calvo, M. Cerrada,

M. Chamizo Llatas, N. Colino, B. De La Cruz, A. Delgado Peris, C. Diez Pardos,

D. Domínguez Vázquez, C. Fernandez Bedoya, J.P. Fernández Ramos, A. Ferrando, J. Flix, M.C. Fouz, P. Garcia-Abia, O. Gonzalez Lopez, S. Goy Lopez, J.M. Hernandez, M.I. Josa, G. Merino, J. Puerta Pelayo, I. Redondo, L. Romero, J. Santaolalla, M.S. Soares, C. Willmott

Universidad Autónoma de Madrid, Madrid, Spain

C. Albajar, G. Codispoti, J.F. de Trocóniz

Universidad de Oviedo, Oviedo, Spain

J. Cuevas, J. Fernandez Menendez, S. Folgueras, I. Gonzalez Caballero, L. Lloret Iglesias, J.M. Vizan Garcia

Instituto de Física de Cantabria (IFCA), CSIC-Universidad de Cantabria, Santander, Spain

J.A. Brochero Cifuentes, I.J. Cabrillo, A. Calderon, S.H. Chuang, J. Duarte Campderros, M. Felcini ${ }^{29}$, M. Fernandez, G. Gomez, J. Gonzalez Sanchez, C. Jorda, P. Lobelle Pardo, A. Lopez Virto, J. Marco, R. Marco, C. Martinez Rivero, F. Matorras, F.J. Munoz Sanchez, J. Piedra Gomez ${ }^{30}$, T. Rodrigo, A.Y. Rodríguez-Marrero, A. Ruiz-Jimeno, L. Scodellaro, M. Sobron Sanudo, I. Vila, R. Vilar Cortabitarte

CERN, European Organization for Nuclear Research, Geneva, Switzerland

D. Abbaneo, E. Auffray, G. Auzinger, P. Baillon, A.H. Ball, D. Barney, C. Bernet ${ }^{5}$,

W. Bialas, P. Bloch, A. Bocci, H. Breuker, K. Bunkowski, T. Camporesi, G. Cerminara,

T. Christiansen, J.A. Coarasa Perez, B. Curé, D. D'Enterria, A. De Roeck, S. Di Guida, M. Dobson, N. Dupont-Sagorin, A. Elliott-Peisert, B. Frisch, W. Funk, A. Gaddi, G. Georgiou, H. Gerwig, M. Giffels, D. Gigi, K. Gill, D. Giordano, M. Giunta, F. Glege, R. GomezReino Garrido, P. Govoni, S. Gowdy, R. Guida, L. Guiducci, S. Gundacker, M. Hansen, C. Hartl, J. Harvey, J. Hegeman, B. Hegner, A. Hinzmann, H.F. Hoffmann, V. Innocente, P. Janot, K. Kaadze, E. Karavakis, K. Kousouris, P. Lecoq, P. Lenzi, C. Lourenço, T. Mäki, 
M. Malberti, L. Malgeri, M. Mannelli, L. Masetti, G. Mavromanolakis, F. Meijers, S. Mersi, E. Meschi, R. Moser, M.U. Mozer, M. Mulders, E. Nesvold, M. Nguyen, T. Orimoto, L. Orsini, E. Palencia Cortezon, E. Perez, A. Petrilli, A. Pfeiffer, M. Pierini, M. Pimiä, D. Piparo, G. Polese, L. Quertenmont, A. Racz, W. Reece, J. Rodrigues Antunes, G. Rolandi ${ }^{31}$, T. Rommerskirchen, C. Rovelli ${ }^{32}$, M. Rovere, H. Sakulin, F. Santanastasio, C. Schäfer, C. Schwick, I. Segoni, A. Sharma, P. Siegrist, P. Silva, M. Simon, P. Sphicas ${ }^{33}$, D. Spiga, M. Spiropulu ${ }^{4}$, M. Stoye, A. Tsirou, G.I. Veres ${ }^{16}$, P. Vichoudis, H.K. Wöhri, S.D. Worm ${ }^{34}$, W.D. Zeuner

\section{Paul Scherrer Institut, Villigen, Switzerland}

W. Bertl, K. Deiters, W. Erdmann, K. Gabathuler, R. Horisberger, Q. Ingram, H.C. Kaestli, S. König, D. Kotlinski, U. Langenegger, F. Meier, D. Renker, T. Rohe, J. Sibille ${ }^{35}$

\section{Institute for Particle Physics, ETH Zurich, Zurich, Switzerland}

L. Bäni, P. Bortignon, M.A. Buchmann, B. Casal, N. Chanon, Z. Chen, S. Cittolin, A. Deisher, G. Dissertori, M. Dittmar, J. Eugster, K. Freudenreich, C. Grab, P. Lecomte, W. Lustermann, P. Martinez Ruiz del Arbol, P. Milenovic ${ }^{36}$, N. Mohr, F. Moortgat, C. Nägeli ${ }^{37}$, P. Nef, F. Nessi-Tedaldi, L. Pape, F. Pauss, M. Peruzzi, F.J. Ronga, M. Rossini, L. Sala, A.K. Sanchez, M.-C. Sawley, A. Starodumov ${ }^{38}$, B. Stieger, M. Takahashi, L. Tauscher ${ }^{\dagger}$, A. Thea, K. Theofilatos, D. Treille, C. Urscheler, R. Wallny, H.A. Weber, L. Wehrli, J. Weng

\section{Universität Zürich, Zurich, Switzerland}

E. Aguilo, C. Amsler, V. Chiochia, S. De Visscher, C. Favaro, M. Ivova Rikova, B. Millan Mejias, P. Otiougova, P. Robmann, A. Schmidt, H. Snoek, M. Verzetti

\section{National Central University, Chung-Li, Taiwan}

Y.H. Chang, K.H. Chen, C.M. Kuo, S.W. Li, W. Lin, Z.K. Liu, Y.J. Lu, D. Mekterovic, R. Volpe, S.S. Yu

\section{National Taiwan University (NTU), Taipei, Taiwan}

P. Bartalini, P. Chang, Y.H. Chang, Y.W. Chang, Y. Chao, K.F. Chen, C. Dietz, U. Grundler, W.-S. Hou, Y. Hsiung, K.Y. Kao, Y.J. Lei, R.-S. Lu, J.G. Shiu, Y.M. Tzeng, X. Wan, M. Wang

\section{Cukurova University, Adana, Turkey}

A. Adiguzel, M.N. Bakirci ${ }^{39}$, S. $\mathrm{Cerci}^{40}$, C. Dozen, I. Dumanoglu, E. Eskut, S. Girgis, G. Gokbulut, I. Hos, E.E. Kangal, G. Karapinar, A. Kayis Topaksu, G. Onengut, K. Ozdemir, S. Ozturk ${ }^{41}$, A. Polatoz, K. Sogut ${ }^{42}$, D. Sunar Cerci ${ }^{40}$, B. Tali ${ }^{40}$, H. Topakli ${ }^{39}$, D. Uzun, L.N. Vergili, M. Vergili

Middle East Technical University, Physics Department, Ankara, Turkey

I.V. Akin, T. Aliev, B. Bilin, S. Bilmis, M. Deniz, H. Gamsizkan, A.M. Guler, K. Ocalan, A. Ozpineci, M. Serin, R. Sever, U.E. Surat, M. Yalvac, E. Yildirim, M. Zeyrek 
Bogazici University, Istanbul, Turkey

M. Deliomeroglu, E. Gülmez, B. Isildak, M. Kaya ${ }^{43}$, O. Kaya ${ }^{43}$, S. Ozkorucuklu ${ }^{44}$, N. Sonmez ${ }^{45}$

National Scientific Center, Kharkov Institute of Physics and Technology, Kharkov, Ukraine

L. Levchuk

University of Bristol, Bristol, United Kingdom

F. Bostock, J.J. Brooke, E. Clement, D. Cussans, H. Flacher, R. Frazier, J. Goldstein, M. Grimes, G.P. Heath, H.F. Heath, L. Kreczko, S. Metson, D.M. Newbold ${ }^{34}$, K. Nirunpong, A. Poll, S. Senkin, V.J. Smith, T. Williams

Rutherford Appleton Laboratory, Didcot, United Kingdom

L. Basso ${ }^{46}$, K.W. Bell, A. Belyaev ${ }^{46}$, C. Brew, R.M. Brown, B. Camanzi, D.J.A. Cockerill, J.A. Coughlan, K. Harder, S. Harper, J. Jackson, B.W. Kennedy, E. Olaiya, D. Petyt, B.C. Radburn-Smith, C.H. Shepherd-Themistocleous, I.R. Tomalin, W.J. Womersley

Imperial College, London, United Kingdom

R. Bainbridge, G. Ball, R. Beuselinck, O. Buchmuller, D. Colling, N. Cripps, M. Cutajar, P. Dauncey, G. Davies, M. Della Negra, W. Ferguson, J. Fulcher, D. Futyan, A. Gilbert, A. Guneratne Bryer, G. Hall, Z. Hatherell, J. Hays, G. Iles, M. Jarvis, G. Karapostoli, L. Lyons, A.-M. Magnan, J. Marrouche, B. Mathias, R. Nandi, J. Nash, A. Nikitenko ${ }^{38}$, A. Papageorgiou, M. Pesaresi, K. Petridis, M. Pioppi ${ }^{47}$, D.M. Raymond, S. Rogerson, N. Rompotis, A. Rose, M.J. Ryan, C. Seez, P. Sharp, A. Sparrow, A. Tapper, S. Tourneur, M. Vazquez Acosta, T. Virdee, S. Wakefield, N. Wardle, D. Wardrope, T. Whyntie

Brunel University, Uxbridge, United Kingdom

M. Barrett, M. Chadwick, J.E. Cole, P.R. Hobson, A. Khan, P. Kyberd, D. Leslie, W. Martin, I.D. Reid, L. Teodorescu, M. Turner

Baylor University, Waco, U.S.A.

K. Hatakeyama, H. Liu, T. Scarborough

The University of Alabama, Tuscaloosa, U.S.A.

C. Henderson

Boston University, Boston, U.S.A.

A. Avetisyan, T. Bose, E. Carrera Jarrin, C. Fantasia, A. Heister, J. St. John, P. Lawson, D. Lazic, J. Rohlf, D. Sperka, L. Sulak

Brown University, Providence, U.S.A.

S. Bhattacharya, D. Cutts, A. Ferapontov, U. Heintz, S. Jabeen, G. Kukartsev, G. Landsberg, M. Luk, M. Narain, D. Nguyen, M. Segala, T. Sinthuprasith, T. Speer, K.V. Tsang

University of California, Davis, Davis, U.S.A.

R. Breedon, G. Breto, M. Calderon De La Barca Sanchez, S. Chauhan, M. Chertok, J. Conway, R. Conway, P.T. Cox, J. Dolen, R. Erbacher, R. Houtz, W. Ko, A. Kopecky, 
R. Lander, O. Mall, T. Miceli, D. Pellett, J. Robles, B. Rutherford, M. Searle, J. Smith, M. Squires, M. Tripathi, R. Vasquez Sierra

University of California, Los Angeles, Los Angeles, U.S.A.

V. Andreev, K. Arisaka, D. Cline, R. Cousins, J. Duris, S. Erhan, P. Everaerts, C. Farrell, J. Hauser, M. Ignatenko, C. Jarvis, C. Plager, G. Rakness, P. Schlein ${ }^{\dagger}$, J. Tucker, V. Valuev, M. Weber

University of California, Riverside, Riverside, U.S.A.

J. Babb, R. Clare, J. Ellison, J.W. Gary, F. Giordano, G. Hanson, G.Y. Jeng, H. Liu, O.R. Long, A. Luthra, H. Nguyen, S. Paramesvaran, J. Sturdy, S. Sumowidagdo, R. Wilken, S. Wimpenny

\section{University of California, San Diego, La Jolla, U.S.A.}

W. Andrews, J.G. Branson, G.B. Cerati, D. Evans, F. Golf, A. Holzner, R. Kelley, M. Lebourgeois, J. Letts, I. Macneill, B. Mangano, S. Padhi, C. Palmer, G. Petrucciani, H. Pi, M. Pieri, R. Ranieri, M. Sani, I. Sfiligoi, V. Sharma, S. Simon, E. Sudano, M. Tadel, Y. Tu, A. Vartak, S. Wasserbaech ${ }^{48}$, F. Würthwein, A. Yagil, J. Yoo

University of California, Santa Barbara, Santa Barbara, U.S.A.

D. Barge, R. Bellan, C. Campagnari, M. D’Alfonso, T. Danielson, K. Flowers, P. Geffert, C. George, J. Incandela, C. Justus, P. Kalavase, S.A. Koay, D. Kovalskyi ${ }^{1}$, V. Krutelyov, S. Lowette, N. Mccoll, S.D. Mullin, V. Pavlunin, F. Rebassoo, J. Ribnik, J. Richman, R. Rossin, D. Stuart, W. To, J.R. Vlimant, C. West

\section{California Institute of Technology, Pasadena, U.S.A.}

A. Apresyan, A. Bornheim, J. Bunn, Y. Chen, E. Di Marco, J. Duarte, M. Gataullin, Y. Ma, A. Mott, H.B. Newman, C. Rogan, V. Timciuc, P. Traczyk, J. Veverka, R. Wilkinson, Y. Yang, R.Y. Zhu

Carnegie Mellon University, Pittsburgh, U.S.A.

B. Akgun, R. Carroll, T. Ferguson, Y. Iiyama, D.W. Jang, S.Y. Jun, Y.F. Liu, M. Paulini, J. Russ, H. Vogel, I. Vorobiev

University of Colorado at Boulder, Boulder, U.S.A.

J.P. Cumalat, M.E. Dinardo, B.R. Drell, C.J. Edelmaier, W.T. Ford, A. Gaz, B. Heyburn, E. Luiggi Lopez, U. Nauenberg, J.G. Smith, K. Stenson, K.A. Ulmer, S.R. Wagner, S.L. Zang

Cornell University, Ithaca, U.S.A.

L. Agostino, J. Alexander, A. Chatterjee, N. Eggert, L.K. Gibbons, B. Heltsley, W. Hopkins, A. Khukhunaishvili, B. Kreis, G. Nicolas Kaufman, J.R. Patterson, D. Puigh, A. Ryd, E. Salvati, X. Shi, W. Sun, W.D. Teo, J. Thom, J. Thompson, J. Vaughan, Y. Weng, L. Winstrom, P. Wittich

Fairfield University, Fairfield, U.S.A.

A. Biselli, G. Cirino, D. Winn 
Fermi National Accelerator Laboratory, Batavia, U.S.A.

S. Abdullin, M. Albrow, J. Anderson, G. Apollinari, M. Atac, J.A. Bakken, L.A.T. Bauerdick, A. Beretvas, J. Berryhill, P.C. Bhat, I. Bloch, K. Burkett, J.N. Butler, V. Chetluru, H.W.K. Cheung, F. Chlebana, S. Cihangir, W. Cooper, D.P. Eartly, V.D. Elvira, S. Esen, I. Fisk, J. Freeman, Y. Gao, E. Gottschalk, D. Green, O. Gutsche, J. Hanlon, R.M. Harris, J. Hirschauer, B. Hooberman, H. Jensen, S. Jindariani, M. Johnson, U. Joshi, B. Klima, S. Kunori, S. Kwan, C. Leonidopoulos, D. Lincoln, R. Lipton, J. Lykken, K. Maeshima, J.M. Marraffino, S. Maruyama, D. Mason, P. McBride, T. Miao, K. Mishra, S. Mrenna, Y. Musienko ${ }^{49}$, C. Newman-Holmes, V. O’Dell, J. Pivarski, R. Pordes, O. Prokofyev, T. Schwarz, E. Sexton-Kennedy, S. Sharma, W.J. Spalding, L. Spiegel, P. Tan, L. Taylor, S. Tkaczyk, L. Uplegger, E.W. Vaandering, R. Vidal, J. Whitmore, W. Wu, F. Yang, F. Yumiceva, J.C. Yun

University of Florida, Gainesville, U.S.A.

D. Acosta, P. Avery, D. Bourilkov, M. Chen, S. Das, M. De Gruttola, G.P. Di Giovanni, D. Dobur, A. Drozdetskiy, R.D. Field, M. Fisher, Y. Fu, I.K. Furic, J. Gartner, S. Goldberg, J. Hugon, B. Kim, J. Konigsberg, A. Korytov, A. Kropivnitskaya, T. Kypreos, J.F. Low, K. Matchev, G. Mitselmakher, L. Muniz, M. Park, R. Remington, A. Rinkevicius, M. Schmitt, B. Scurlock, P. Sellers, N. Skhirtladze, M. Snowball, D. Wang, J. Yelton, M. Zakaria

Florida International University, Miami, U.S.A.

V. Gaultney, L.M. Lebolo, S. Linn, P. Markowitz, G. Martinez, J.L. Rodriguez

Florida State University, Tallahassee, U.S.A.

T. Adams, A. Askew, J. Bochenek, J. Chen, B. Diamond, S.V. Gleyzer, J. Haas, S. Hagopian, V. Hagopian, M. Jenkins, K.F. Johnson, H. Prosper, S. Sekmen, V. Veeraraghavan, M. Weinberg

Florida Institute of Technology, Melbourne, U.S.A.

M.M. Baarmand, B. Dorney, M. Hohlmann, H. Kalakhety, I. Vodopiyanov

University of Illinois at Chicago (UIC), Chicago, U.S.A.

M.R. Adams, I.M. Anghel, L. Apanasevich, Y. Bai, V.E. Bazterra, R.R. Betts, J. Callner, R. Cavanaugh, C. Dragoiu, L. Gauthier, C.E. Gerber, D.J. Hofman, S. Khalatyan, G.J. Kunde ${ }^{50}$, F. Lacroix, M. Malek, C. O'Brien, C. Silkworth, C. Silvestre, D. Strom, N. Varelas

\section{The University of Iowa, Iowa City, U.S.A.}

U. Akgun, E.A. Albayrak, B. Bilki, W. Clarida, F. Duru, S. Griffiths, C.K. Lae, E. McCliment, J.-P. Merlo, H. Mermerkaya ${ }^{51}$, A. Mestvirishvili, A. Moeller, J. Nachtman, C.R. Newsom, E. Norbeck, J. Olson, Y. Onel, F. Ozok, S. Sen, E. Tiras, J. Wetzel, T. Yetkin, K. Yi

Johns Hopkins University, Baltimore, U.S.A.

B.A. Barnett, B. Blumenfeld, S. Bolognesi, A. Bonato, C. Eskew, D. Fehling, G. Giurgiu, A.V. Gritsan, Z.J. Guo, G. Hu, P. Maksimovic, S. Rappoccio, M. Swartz, N.V. Tran, A. Whitbeck 
The University of Kansas, Lawrence, U.S.A.

P. Baringer, A. Bean, G. Benelli, O. Grachov, R.P. Kenny Iii, M. Murray, D. Noonan, S. Sanders, R. Stringer, G. Tinti, J.S. Wood, V. Zhukova

Kansas State University, Manhattan, U.S.A.

A.F. Barfuss, T. Bolton, I. Chakaberia, A. Ivanov, S. Khalil, M. Makouski, Y. Maravin, S. Shrestha, I. Svintradze

Lawrence Livermore National Laboratory, Livermore, U.S.A.

J. Gronberg, D. Lange, D. Wright

University of Maryland, College Park, U.S.A.

A. Baden, M. Boutemeur, B. Calvert, S.C. Eno, J.A. Gomez, N.J. Hadley, R.G. Kellogg, M. Kirn, Y. Lu, A.C. Mignerey, A. Peterman, K. Rossato, P. Rumerio, A. Skuja, J. Temple, M.B. Tonjes, S.C. Tonwar, E. Twedt

Massachusetts Institute of Technology, Cambridge, U.S.A.

B. Alver, G. Bauer, J. Bendavid, W. Busza, E. Butz, I.A. Cali, M. Chan, V. Dutta, G. Gomez Ceballos, M. Goncharov, K.A. Hahn, P. Harris, Y. Kim, M. Klute, Y.-J. Lee, W. Li, P.D. Luckey, T. Ma, S. Nahn, C. Paus, D. Ralph, C. Roland, G. Roland, M. Rudolph, G.S.F. Stephans, F. Stöckli, K. Sumorok, K. Sung, D. Velicanu, E.A. Wenger, R. Wolf, B. Wyslouch, S. Xie, M. Yang, Y. Yilmaz, A.S. Yoon, M. Zanetti

University of Minnesota, Minneapolis, U.S.A.

S.I. Cooper, P. Cushman, B. Dahmes, A. De Benedetti, G. Franzoni, A. Gude, J. Haupt, S.C. Kao, K. Klapoetke, Y. Kubota, J. Mans, N. Pastika, V. Rekovic, R. Rusack, M. Sasseville, A. Singovsky, N. Tambe, J. Turkewitz

University of Mississippi, University, U.S.A.

L.M. Cremaldi, R. Godang, R. Kroeger, L. Perera, R. Rahmat, D.A. Sanders, D. Summers

University of Nebraska-Lincoln, Lincoln, U.S.A.

E. Avdeeva, K. Bloom, S. Bose, J. Butt, D.R. Claes, A. Dominguez, M. Eads, P. Jindal, J. Keller, I. Kravchenko, J. Lazo-Flores, H. Malbouisson, S. Malik, G.R. Snow

State University of New York at Buffalo, Buffalo, U.S.A.

U. Baur, A. Godshalk, I. Iashvili, S. Jain, A. Kharchilava, A. Kumar, S.P. Shipkowski, K. Smith, Z. Wan

\section{Northeastern University, Boston, U.S.A.}

G. Alverson, E. Barberis, D. Baumgartel, M. Chasco, D. Trocino, D. Wood, J. Zhang

Northwestern University, Evanston, U.S.A.

A. Anastassov, A. Kubik, N. Mucia, N. Odell, R.A. Ofierzynski, B. Pollack, A. Pozdnyakov, M. Schmitt, S. Stoynev, M. Velasco, S. Won 
University of Notre Dame, Notre Dame, U.S.A.

L. Antonelli, D. Berry, A. Brinkerhoff, M. Hildreth, C. Jessop, D.J. Karmgard, J. Kolb,

T. Kolberg, K. Lannon, W. Luo, S. Lynch, N. Marinelli, D.M. Morse, T. Pearson, R. Ruchti,

J. Slaunwhite, N. Valls, M. Wayne, M. Wolf, J. Ziegler

The Ohio State University, Columbus, U.S.A.

B. Bylsma, L.S. Durkin, C. Hill, P. Killewald, K. Kotov, T.Y. Ling, M. Rodenburg, C. Vuosalo, G. Williams

Princeton University, Princeton, U.S.A.

N. Adam, E. Berry, P. Elmer, D. Gerbaudo, V. Halyo, P. Hebda, A. Hunt, E. Laird,

D. Lopes Pegna, P. Lujan, D. Marlow, T. Medvedeva, M. Mooney, J. Olsen, P. Piroué,

X. Quan, A. Raval, H. Saka, D. Stickland, C. Tully, J.S. Werner, A. Zuranski

University of Puerto Rico, Mayaguez, U.S.A.

J.G. Acosta, X.T. Huang, A. Lopez, H. Mendez, S. Oliveros, J.E. Ramirez Vargas, A. Zatserklyaniy

Purdue University, West Lafayette, U.S.A.

E. Alagoz, V.E. Barnes, D. Benedetti, G. Bolla, L. Borrello, D. Bortoletto, M. De Mattia, A. Everett, L. Gutay, Z. Hu, M. Jones, O. Koybasi, M. Kress, A.T. Laasanen, N. Leonardo, V. Maroussov, P. Merkel, D.H. Miller, N. Neumeister, I. Shipsey, D. Silvers, A. Svyatkovskiy, M. Vidal Marono, H.D. Yoo, J. Zablocki, Y. Zheng

Purdue University Calumet, Hammond, U.S.A.

S. Guragain, N. Parashar

Rice University, Houston, U.S.A.

A. Adair, C. Boulahouache, V. Cuplov, K.M. Ecklund, F.J.M. Geurts, B.P. Padley, R. Redjimi, J. Roberts, J. Zabel

University of Rochester, Rochester, U.S.A.

B. Betchart, A. Bodek, Y.S. Chung, R. Covarelli, P. de Barbaro, R. Demina, Y. Eshaq, A. Garcia-Bellido, P. Goldenzweig, Y. Gotra, J. Han, A. Harel, D.C. Miner, G. Petrillo, W. Sakumoto, D. Vishnevskiy, M. Zielinski

The Rockefeller University, New York, U.S.A.

A. Bhatti, R. Ciesielski, L. Demortier, K. Goulianos, G. Lungu, S. Malik, C. Mesropian

Rutgers, the State University of New Jersey, Piscataway, U.S.A.

S. Arora, O. Atramentov, A. Barker, J.P. Chou, C. Contreras-Campana, E. ContrerasCampana, D. Duggan, D. Ferencek, Y. Gershtein, R. Gray, E. Halkiadakis, D. Hidas, D. Hits, A. Lath, S. Panwalkar, M. Park, R. Patel, A. Richards, K. Rose, S. Salur, S. Schnetzer, S. Somalwar, R. Stone, S. Thomas

University of Tennessee, Knoxville, U.S.A.

G. Cerizza, M. Hollingsworth, S. Spanier, Z.C. Yang, A. York 


\section{Texas A\&M University, College Station, U.S.A.}

R. Eusebi, W. Flanagan, J. Gilmore, T. Kamon ${ }^{52}$, V. Khotilovich, R. Montalvo, I. Osipenkov, Y. Pakhotin, A. Perloff, J. Roe, A. Safonov, S. Sengupta, I. Suarez, A. Tatarinov, D. Toback

\section{Texas Tech University, Lubbock, U.S.A.}

N. Akchurin, C. Bardak, J. Damgov, P.R. Dudero, C. Jeong, K. Kovitanggoon, S.W. Lee, T. Libeiro, P. Mane, Y. Roh, A. Sill, I. Volobouev, R. Wigmans, E. Yazgan

\section{Vanderbilt University, Nashville, U.S.A.}

E. Appelt, E. Brownson, D. Engh, C. Florez, W. Gabella, A. Gurrola, M. Issah, W. Johns, C. Johnston, P. Kurt, C. Maguire, A. Melo, P. Sheldon, B. Snook, S. Tuo, J. Velkovska

\section{University of Virginia, Charlottesville, U.S.A.}

M.W. Arenton, M. Balazs, S. Boutle, S. Conetti, B. Cox, B. Francis, S. Goadhouse, J. Goodell, R. Hirosky, A. Ledovskoy, C. Lin, C. Neu, J. Wood, R. Yohay

\section{Wayne State University, Detroit, U.S.A.}

S. Gollapinni, R. Harr, P.E. Karchin, C. Kottachchi Kankanamge Don, P. Lamichhane, M. Mattson, C. Milstène, A. Sakharov

\section{University of Wisconsin, Madison, U.S.A.}

M. Anderson, M. Bachtis, D. Belknap, J.N. Bellinger, J. Bernardini, D. Carlsmith, M. Cepeda, S. Dasu, J. Efron, E. Friis, L. Gray, K.S. Grogg, M. Grothe, R. Hall-Wilton, M. Herndon, A. Hervé, P. Klabbers, J. Klukas, A. Lanaro, C. Lazaridis, J. Leonard, R. Loveless, A. Mohapatra, I. Ojalvo, G.A. Pierro, I. Ross, A. Savin, W.H. Smith, J. Swanson

$\dagger$ : Deceased

1: Also at CERN, European Organization for Nuclear Research, Geneva, Switzerland

2: Also at National Institute of Chemical Physics and Biophysics, Tallinn, Estonia

3: Also at Universidade Federal do ABC, Santo Andre, Brazil

4: Also at California Institute of Technology, Pasadena, U.S.A.

5: Also at Laboratoire Leprince-Ringuet, Ecole Polytechnique, IN2P3-CNRS, Palaiseau, France

6: Also at Suez Canal University, Suez, Egypt

7: Also at Cairo University, Cairo, Egypt

8: Also at British University, Cairo, Egypt

9: Also at Fayoum University, El-Fayoum, Egypt

10: Also at Ain Shams University, Cairo, Egypt

11: Also at Soltan Institute for Nuclear Studies, Warsaw, Poland

12: Also at Université de Haute-Alsace, Mulhouse, France

13: Also at Moscow State University, Moscow, Russia

14: Also at Brandenburg University of Technology, Cottbus, Germany

15: Also at Institute of Nuclear Research ATOMKI, Debrecen, Hungary

16: Also at Eötvös Loránd University, Budapest, Hungary

17: Also at Tata Institute of Fundamental Research - HECR, Mumbai, India

18: Now at King Abdulaziz University, Jeddah, Saudi Arabia

19: Also at University of Visva-Bharati, Santiniketan, India 
20: Also at Sharif University of Technology, Tehran, Iran

21: Also at Isfahan University of Technology, Isfahan, Iran

22: Also at Shiraz University, Shiraz, Iran

23: Also at Plasma Physics Research Center, Science and Research Branch, Islamic Azad University, Teheran, Iran

24: Also at Facoltà Ingegneria Università di Roma, Roma, Italy

25: Also at Università della Basilicata, Potenza, Italy

26: Also at Laboratori Nazionali di Legnaro dell' INFN, Legnaro, Italy

27: Also at Università degli studi di Siena, Siena, Italy

28: Also at Faculty of Physics of University of Belgrade, Belgrade, Serbia

29: Also at University of California, Los Angeles, Los Angeles, U.S.A.

30: Also at University of Florida, Gainesville, U.S.A.

31: Also at Scuola Normale e Sezione dell' INFN, Pisa, Italy

32: Also at INFN Sezione di Roma; Università di Roma "La Sapienza", Roma, Italy

33: Also at University of Athens, Athens, Greece

34: Also at Rutherford Appleton Laboratory, Didcot, United Kingdom

35: Also at The University of Kansas, Lawrence, U.S.A.

36: Also at University of Belgrade, Faculty of Physics and Vinca Institute of Nuclear Sciences, Belgrade, Serbia

37: Also at Paul Scherrer Institut, Villigen, Switzerland

38: Also at Institute for Theoretical and Experimental Physics, Moscow, Russia

39: Also at Gaziosmanpasa University, Tokat, Turkey

40: Also at Adiyaman University, Adiyaman, Turkey

41: Also at The University of Iowa, Iowa City, U.S.A.

42: Also at Mersin University, Mersin, Turkey

43: Also at Kafkas University, Kars, Turkey

44: Also at Suleyman Demirel University, Isparta, Turkey

45: Also at Ege University, Izmir, Turkey

46: Also at School of Physics and Astronomy, University of Southampton, Southampton, United Kingdom

47: Also at INFN Sezione di Perugia; Università di Perugia, Perugia, Italy

48: Also at Utah Valley University, Orem, U.S.A.

49: Also at Institute for Nuclear Research, Moscow, Russia

50: Also at Los Alamos National Laboratory, Los Alamos, U.S.A.

51: Also at Erzincan University, Erzincan, Turkey

52: Also at Kyungpook National University, Daegu, Korea 